\title{
Variational modelling of wave-structure interactions with an offshore wind-turbine mast
}

\author{
Tomasz Salwa • Onno Bokhove • \\ Mark A. Kelmanson
}

Received: 28 February 2017 / Accepted: 8 August 2017 / Published online: 15 September 2017

(C) The Author(s) 2017. This article is an open access publication

\begin{abstract}
We consider the development of a mathematical model of water waves interacting with the mast of an offshore wind turbine. A variational approach is used for which the starting point is an action functional describing a dual system comprising a potential-flow fluid, a solid structure modelled with nonlinear elasticity, and the coupling between them. We develop a linearized model of the fluid-structure or wave-mast coupling, which is a linearization of the variational principle for the fully coupled nonlinear model. Our numerical results for the linear case indicate that our variational approach yields a stable numerical discretization of a fully coupled model of water waves and an elastic beam. The energy exchange between the subsystems is seen to be in balance, yielding a total energy that shows only small and bounded oscillations amplitude of which tends to zero with the second-order convergence as the timestep approaches zero. Similar second-order convergence is observed for spatial mesh refinement. The linearized model so far developed can be extended to a nonlinear regime.
\end{abstract}

Keywords Computational fluid dynamics · Fluid-structure interaction · Hamiltonian mechanics · Potential flow · Variational principle $\cdot$ Water waves

\section{Introduction}

The search for alternative and effective sustainable energy sources that support balanced growth has led to an increased focus on offshore wind energy. Both visibility issues and wind supply play major roles in the development of this particular branch of wind energy. There are two main directions of active research in this field, namely offshore floating platforms with wind turbines and fixed-bottom monopile wind farms in shallow water: a review is given in [1]. The first branch is still in the prototype stage of development. The latter branch, i.e. concerning shallow and

T. Salwa $(\varangle) \cdot$ O. Bokhove · M. A. Kelmanson

School of Mathematics, University of Leeds, Leeds LS2 9JT, UK

e-mail: mmtjs@leeds.ac.uk

O. Bokhove

e-mail: o.bokhove@leeds.ac.uk

M. A. Kelmanson

e-mail: m.kelmanson@leeds.ac.uk 


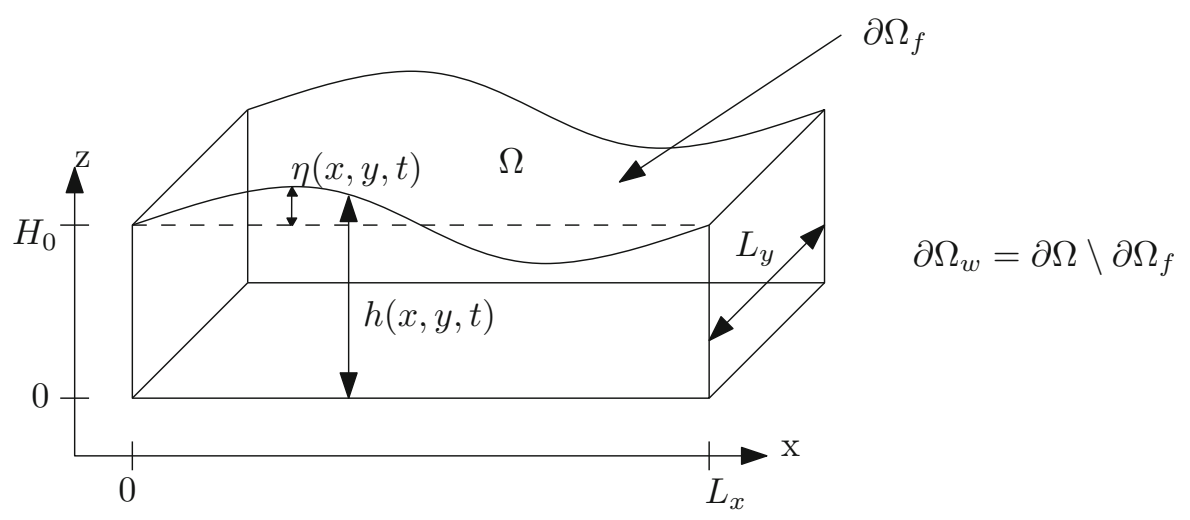

Fig. 1 Geometry of the fluid domain: a box with rest-state dimensions $L_{x} \times L_{y} \times H_{0}$ and evolving free surface $z=h(x, y, t)$. Here $\eta(x, y, t)$ is the free-surface perturbation from the rest state that first appears in (26)

intermediate-depth water, fixed-bottom wind turbines, already exists, e.g. in areas of the North Sea. It is accordingly considered here.

Ultimately, we aim to develop a mathematical model of wave impact on a single beam $/ \mathrm{mast}$ of a wind turbine, with particular emphasis on breaking waves. Similar models were considered in [2,3]. One way to proceed is to incorporate the water and air phases as a mixture that arises during wave breaking. We have earlier shown [4] that this mixture model reduces to a standard potential-flow model in the event that the phases are separated and the waves do not break. The latter, reduced case constitutes a developmental check since the potential-flow model for water waves is an industrial and mathematical benchmark.

The model developed herein can also include the beam as an integral part of the coupled fluid-structure interaction (FSI). The FSI problems are known to suffer from numerical instabilities [1]. Our method is based on reduction of the whole system to an abstract Hamiltonian form, to which known stable discrete schemes can be applied. After returning to the original variables, the scheme remains stable by construction; we shall show that it results in the addition of novel regularization terms due to the fluid-beam coupling.

As a starting point, we therefore consider a variational principle (VP) for surface gravity waves coupled to a nonlinear elastic beam, using [5-7]. The advantage of this approach is that the whole system is described by a single VP that is discretized directly in both space and time. This ensures stability and overall energy conservation. Variation of this algebraic VP yields the so-called Galerkin finite-element model, with mixed dis/continuous element approximations [8]. The FSI is embedded in a single variational formulation with the associated conservative properties akin to the ones in the parent continuum system. Our numerical results for the linearized system indicate that our approach by construction yields, as anticipated, a stable numerical scheme.

The paper is organized as follows. Section 2 describes the formulation of the model. First, the VP for the potential flow is introduced. Second the same is shown for the nonlinear elastic beam model, which is subsequently linearized. Third, the model of a fully coupled fluid-beam system is presented. Finally, we proceed with its linearization. Section 3 describes the solution of the linear model. First, the Finite-Element Method (FEM) is used to discretize the system in space. Second, the system is reduced to Hamiltonian form and temporal discretization is applied. Section 4 presents not only 2D results of the code written in plain python, but also 3D results obtained using the FEM automation system Firedrake [9]. Section 5 concludes the paper. Although some elements of nascent aspects of this work have been presented in offshore-engineering conferences [10,11], this paper provides full details of the derivation and implementation of the coupled linear beam-fluid system, and of the modelling of the coupled non-linear beam-fluid system. 


\section{Nonlinear variational formulation}

\subsection{Potential-flow water waves}

We consider water as an incompressible fluid with density $\rho$. The vector velocity field $\mathbf{u}=\mathbf{u}(x, y, z, t)$ has zero divergence, $\boldsymbol{\nabla} \cdot \mathbf{u}=0$, with spatial coordinates $\mathbf{x}=(x, y, z)^{T}$ and time coordinate $t$. Gravity acts in the negative $z$-direction and the associated acceleration of gravity is $g$. The velocity is expressed in terms of a scalar potential $\phi=\phi(x, y, z, t)$ such that $\mathbf{u}=\nabla \phi$. We consider flow in the 3D Cartesian domain $\Omega$ (see Fig. 1) bounded by solid walls at $x=0, x=L_{x}, y=0, y=L_{y}$ and the flat bottom at $z=0$. The upper surface of $\Omega$ is given by the single-valued evolving free surface $z=h(x, y, t)$, and hence $\Omega=\left[0, L_{x}\right] \times\left[0, L_{y}\right] \times[0, h(x, y, t)]$, within which Luke's [6] VP for potential-flow water waves reads

$$
\begin{aligned}
0 & =\delta \int_{0}^{T} \iiint_{\Omega}-\rho \partial_{t} \phi \mathrm{d} \Omega-\mathcal{H} \mathrm{d} t \\
& \equiv \delta \int_{0}^{T} \int_{0}^{L_{x}} \int_{0}^{L_{y}} \int_{0}^{h(x, y, t)}-\rho\left(\partial_{t} \phi+\frac{1}{2}|\nabla \phi|^{2}+g\left(z-H_{0}\right)\right) \mathrm{d} z \mathrm{~d} y \mathrm{~d} x \mathrm{~d} t,
\end{aligned}
$$

in which $H_{0}$ is the rest-state water level. The energy or Hamiltonian $\mathcal{H}$ consists of the sum of kinetic and potential energies. We use integration by parts in time together with Gauss' law with outward normal $\mathbf{n}=$ $\left(-\nabla_{\perp} h, 1\right)^{T} / \sqrt{1+\left|\nabla_{\perp} h\right|^{2}}$ at the free surface, in which $\nabla_{\perp}=\left(\partial_{x}, \partial_{y}\right)$. The passive and constant air pressure is denoted by $p_{a}$. Then, variation of (1) yields

$$
\begin{aligned}
0= & \int_{0}^{T} \int_{0}^{L_{x}} \int_{0}^{L_{y}} \int_{0}^{h(x, y, t)} \rho \nabla^{2} \phi \delta \phi \mathrm{d} z \mathrm{~d} y \mathrm{~d} x-\int_{\partial \Omega_{w}} \rho \nabla \phi \cdot \mathbf{n} \delta \phi \mathrm{d} S \\
& +\left.\left.\int_{0}^{L_{x}} \int_{0}^{L_{y}} \rho\left(-\partial_{z} \phi+\partial_{x} \phi \partial_{x} h+\partial_{y} \phi \partial_{y} h+\partial_{t} h\right)\right|_{z=h} \delta \phi\right|_{z=h}+\left(p-p_{a}\right)_{z=h} \delta h \mathrm{~d} y \mathrm{~d} x \mathrm{~d} t,
\end{aligned}
$$

in which the pressure difference $p-p_{a}$ here acts as a shorthand placeholder for the Bernoulli expression $-\rho\left(\partial_{t} \phi+\frac{1}{2}|\nabla \phi|^{2}+g\left(z-H_{0}\right)\right)$.

The equations of motion emerge from relation (2), augmented by the following non-normal-flow boundary conditions $\nabla \phi \cdot \mathbf{n}=0$, with unit outward normal $\mathbf{n}$ at solid walls $\partial \Omega_{w}$

$$
\begin{array}{lrl}
x \in\left[0, L_{x}\right], y \in\left[0, L_{y}\right], z \in[0, h] ; & \delta \phi: 0=-\rho \nabla^{2} \phi=\frac{\delta \mathcal{H}}{\delta \phi}, \\
x \in\left[0, L_{x}\right], y \in\left[0, L_{y}\right], z=h(x, y, t) ; & (\delta \phi)_{h}: \partial_{t} h=-\partial_{x} \phi \partial_{x} h+\partial_{z} \phi=\frac{\delta \mathcal{H}}{(\delta \phi)_{h}}, \\
x \in\left[0, L_{x}\right], y \in\left[0, L_{y}\right], z=h(x, y, t) ; & \delta h: \rho \partial_{t} \phi=-\frac{1}{2} \rho|\nabla \phi|^{2}-\rho g\left(h-H_{0}\right)=-\frac{\delta \mathcal{H}}{\delta h} .
\end{array}
$$

The above equations can be extended to include a wavemaker.

\subsection{Geometrically nonlinear elastic mast}

We consider a nonlinear hyperelastic model for an elastic material in which the geometric nonlinearity of the displacements is also taken into account. The constitutive law is such that, after linearization, it satisfies a linear Hooke's law. The choice of this model is guided by our goal of coupling the potential-flow water-wave model to a weakly nonlinear elastic model.

We first model the positions $\mathbf{X}=\mathbf{X}(a, b, c, t)=(X, Y, Z)^{T}=\left(X_{1}, X_{2}, X_{3}\right)^{T}$ of an infinitesimal 3D element of solid material as a function of Lagrangian coordinates $\mathbf{a}=(a, b, c)^{T}=\left(a_{1}, a_{2}, a_{3}\right)^{T}$ in the reference domain $\Omega_{0}$ 


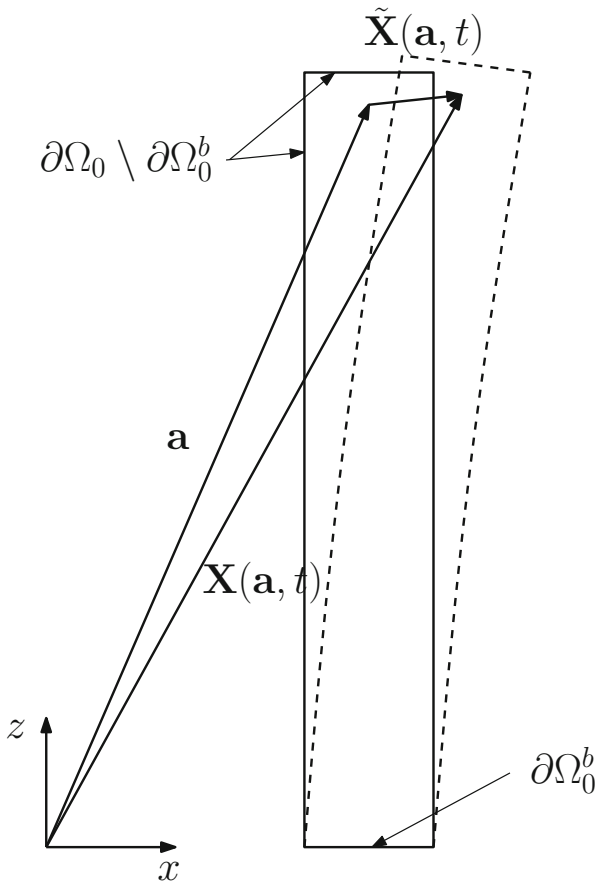

Fig. 2 Sketch of the beam geometry, depicting a cross-section in the $x-z$ plane, in which $\mathbf{a}=\mathbf{X}(\mathbf{a}, 0)$ is the Lagrangian coordinate in the reference state (solid boundary). $\mathbf{X}(\mathbf{a}, t)$ is the position of a point in the shifted beam (dashed boundary) and $\tilde{\mathbf{X}}(\mathbf{a}, t)$ its deflection, $\partial \Omega_{0}$ denotes the structure boundary and $\partial \Omega_{0}^{b}$ its fixed bottom

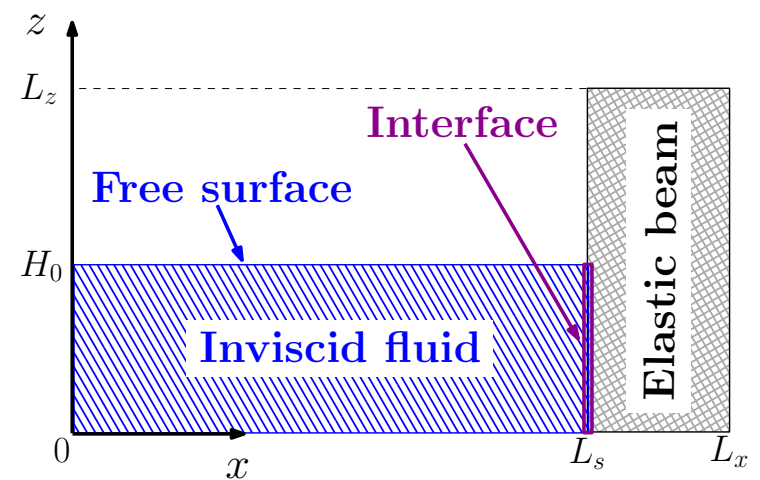

Fig. 3 Geometry of the linearized or rest system: fluid (hatched) and elastic beam (cross-hatched). This 2D representation is in the $y=0$ plane, with the $y$-axis directed into the page, in which direction the full 3D configuration has uniform depth $L_{y}$

with boundary $\partial \Omega_{0}$ and time $t$. At time $t=0$, we take $\mathbf{X}(\mathbf{a}, 0)=\mathbf{a}$, see Fig. 2. The displacements $\tilde{\mathbf{X}}$ follow from the positions as $\tilde{\mathbf{X}}=\mathbf{X}-\mathbf{a}$. The velocity of the displacements is $\partial_{t} \tilde{\mathbf{X}}=\partial_{t} \mathbf{X}=\mathbf{U}=(U, V, W)^{T}=\left(U_{1}, U_{2}, U_{3}\right)^{T}$, where the displacement velocity $\mathbf{U}=\mathbf{U}(\mathbf{a}, t)$ is again a function of Lagrangian coordinates a and time $t$. The variational formulation of the elastic material follows closely the variational formulation of a linear elastic solid obeying Hooke's law, i.e., the constitutive model is linear. However, the geometry is nonlinear as the material is Lagrangian with finite, rather than infinitesimal, displacements. The variational formulation then comprises the kinetic and potential energies in the Lagrangian framework, so that the VP for the hyperelastic model from [12], in a format adjusted to our present purposes, becomes

$0=\delta \int_{0}^{T} \iiint_{\Omega_{0}} \rho_{0} \partial_{t} \mathbf{X} \cdot \mathbf{U}-\frac{1}{2} \rho_{0}|\mathbf{U}|^{2}-\rho_{0} g Z-\frac{1}{2} \lambda[\operatorname{tr}(\mathbf{E})]^{2}-\mu \operatorname{tr}\left(\mathbf{E}^{2}\right) \mathrm{d} a \mathrm{~d} b \mathrm{~d} c \mathrm{~d} t$,

in which $\rho_{0}$ is the uniform material density, $\lambda$ and $\mu$ are respectively the first and second Lamé constants, and $\mathbf{E}$ is given by

$\mathbf{E}=\frac{1}{2}\left(\mathbf{F}^{T} \cdot \mathbf{F}-\mathbf{I}\right)$

where $\mathbf{I}$ is the identity matrix and in which the matrix $\mathbf{F}$, given by 
$\mathbf{F}=\frac{\partial \mathbf{X}}{\partial \mathbf{a}}=\frac{\partial(X, Y, Z)}{\partial(a, b, c)}, \quad$ or equivalently $\quad F_{i j}=\frac{\partial X_{i}}{\partial a_{j}}, \quad$ for $i, j=1,2,3$,

yields the determinant $J$ between the Eulerian and Lagrangian frameworks that accounts for the geometric nonlinearity. The determinant $J$ is given explicitly by

$$
\begin{aligned}
J & =\operatorname{det}(\mathbf{F}) \equiv\left|\frac{\partial(X, Y, Z)}{\partial(a, b, c)}\right| \\
& =X_{a} Y_{b} Z_{c}+Y_{a} Z_{b} X_{c}+Z_{a} X_{b} Y_{c}-X_{c} Y_{b} Z_{a}-Y_{c} Z_{b} X_{a}-Z_{c} X_{b} Y_{a},
\end{aligned}
$$

with subscripts denoting $X_{a} \equiv \partial_{a} X$, et cetera. We model a beam fixed at the bottom $\partial \Omega_{0}^{b}$, so that $\mathbf{X}(a, b, 0, t)=\mathbf{0}$ and $\mathbf{U}(a, b, 0, t)=\mathbf{0}$, which implies that $\left.\delta \mathbf{X}\right|_{\partial \Omega_{0}^{b}}=\mathbf{0}$ and $\left.\delta \mathbf{U}\right|_{\partial \Omega_{0}^{b}}=\mathbf{0}$. Thus, evaluation of the variation in (4) yields

$$
\begin{aligned}
0= & \delta \int_{0}^{T} \iiint_{\Omega_{0}} \rho_{0}\left(\partial_{t} \mathbf{X}-\mathbf{U}\right) \cdot \delta \mathbf{U}-\rho_{0} \partial_{t} \mathbf{U} \cdot \delta \mathbf{X}-\rho_{0} \delta_{l 3} \delta X_{l} \\
& +\partial_{a_{i}}\left(\lambda \operatorname{tr}(\mathbf{E}) F_{l i}+2 \mu E_{k i} F_{l k}\right) \delta X_{l} \mathrm{~d} a \mathrm{~d} b \mathrm{~d} c \\
& -\iint_{\partial \Omega_{0} \backslash \partial \Omega_{0}^{b}} n_{i}\left(\lambda \operatorname{tr}(\mathbf{E}) F_{l i}+2 \mu E_{k i} F_{l k}\right) \delta X_{l} \mathrm{~d} S \mathrm{~d} t,
\end{aligned}
$$

in which we have used the temporal end-point conditions $\delta \mathbf{X}(0)=\delta \mathbf{X}(T)=\mathbf{0}$, as well as, from (5),

$$
E_{i j}=\frac{1}{2}\left(F_{k i} F_{k j}-\delta_{i j}\right)=E_{j i} \text { and } \delta E_{i j}=\frac{1}{2}\left(F_{k i} \delta F_{k j}+F_{k i} \delta F_{k j}\right)
$$

Given the arbitrariness of the respective variations, the resulting equations of motion become

$$
\begin{array}{cl}
\delta \mathbf{U}: & \partial_{t} \mathbf{X}=\mathbf{U} \quad \text { in } \Omega_{0}, \\
\delta X_{l}: & \rho_{0} \partial_{t} U_{l}=-\rho_{0} g \delta_{3 l}+\partial_{a_{i}}\left(\lambda \operatorname{tr}(\mathbf{E}) F_{l i}+2 \mu E_{k i} F_{l k}\right) \\
=-\rho_{0} g \delta_{3 l}+\partial_{a_{i}} T_{l i} \text { in } \Omega_{0}, \\
\delta X_{l}: & 0=n_{i}\left(\lambda \operatorname{tr}(\mathbf{E}) F_{l i}+2 \mu E_{k i} F_{l k}\right)=n_{i} T_{l i} \text { on } \partial \Omega_{0} \backslash \partial \Omega_{0}^{b}
\end{array}
$$

with stress tensor $T_{l i}=\lambda \operatorname{tr}(\mathbf{E}) F_{l i}+2 \mu E_{k i} F_{l k}$.

\subsection{Linearized elastic dynamics}

We proceed with the linearization of (4), together with the transformation from a Lagrangian to an Eulerian description. Since we are ultimately interested in the dynamics of the fluid-structure interaction, we neglect the gravity term. Given (see Fig. 2) that $\mathbf{X}=\mathbf{a}+\tilde{\mathbf{X}}$, we find that (5) can be written as [13]

$\mathbf{E}=\frac{1}{2}\left(\left(\frac{\partial \tilde{\mathbf{X}}}{\partial \mathbf{a}}\right)^{T}+\left(\frac{\partial \tilde{\mathbf{X}}}{\partial \mathbf{a}}\right)\right)+\frac{1}{2}\left(\frac{\partial \tilde{\mathbf{X}}}{\partial \mathbf{a}}\right)^{T} \cdot\left(\frac{\partial \tilde{\mathbf{X}}}{\partial \mathbf{a}}\right)$.

The linearization entails assuming that the displacement gradient is small compared to unity, i.e., $\left\|\frac{\partial \tilde{\mathbf{X}}}{\partial a}\right\| \ll 1$, so that second- and higher-order terms can be neglected. Therefore, the linearized version $\mathbf{e}$ of $\mathbf{E}$ is [13] 
$\mathbf{e}=\frac{1}{2}\left(\left(\frac{\partial \tilde{\mathbf{X}}}{\partial \mathbf{a}}\right)^{T}+\left(\frac{\partial \tilde{\mathbf{X}}}{\partial \mathbf{a}}\right)\right) \quad$ or $\quad e_{i j}=\frac{1}{2}\left(\frac{\partial \tilde{X}_{j}}{\partial a_{i}}+\frac{\partial \tilde{X}_{i}}{\partial a_{j}}\right)$.

Moreover, $\operatorname{tr}(\mathbf{E})^{2}=E_{i i} E_{j j} \approx e_{i i} e_{j j}$ and $\operatorname{tr}(\mathbf{E} \cdot \mathbf{E})=E_{i j}^{2} \approx e_{i j}^{2}$, whence (4) becomes

$0=\delta \int_{0}^{T} \iiint_{\Omega_{0}} \rho_{0} \partial_{t} \tilde{\mathbf{X}} \cdot \mathbf{U}-\frac{1}{2} \rho_{0}|\mathbf{U}|^{2}-\frac{1}{2} \lambda e_{i i} e_{j j}-\mu e_{i j}^{2} \mathrm{~d} a \mathrm{~d} b \mathrm{~d} c \mathrm{~d} t$.

Since the fluid is described in the Eulerian framework, it is useful to work in the same coordinates with the structure. Therefore, we transform (13) to Eulerian coordinates. For clarity, functions in Eulerian coordinates are temporarily annotated with a superscript $(\cdot)^{E}$ so that $f(\mathbf{a})=f^{E}(\mathbf{x}=\mathbf{X}(\mathbf{a}))$. First, since $\mathbf{x}=\mathbf{X}(\mathbf{a}, t)$ and $\mathbf{X}=\mathbf{a}+\tilde{\mathbf{X}}$, we note that

$\frac{\partial \tilde{\mathbf{X}}}{\partial \mathbf{a}}=\frac{\partial \mathbf{X}}{\partial \mathbf{a}} \frac{\partial \tilde{\mathbf{X}}^{E}}{\partial \mathbf{x}}=\left(\mathbf{I}+\frac{\partial \tilde{\mathbf{X}}}{\partial \mathbf{a}}\right) \frac{\partial \tilde{\mathbf{X}}^{E}}{\partial \mathbf{x}}$,

and hence

$$
\frac{\partial \tilde{\mathbf{X}}}{\partial \mathbf{a}}=\left(\mathbf{I}-\frac{\partial \tilde{\mathbf{X}}^{E}}{\partial \mathbf{x}}\right)^{-1} \frac{\partial \tilde{\mathbf{X}}^{E}}{\partial \mathbf{x}} \approx \frac{\partial \tilde{\mathbf{X}}^{E}}{\partial \mathbf{x}}
$$

and

$\mathbf{e} \approx \frac{1}{2}\left(\left(\frac{\partial \tilde{\mathbf{X}}^{E}}{\partial \mathbf{x}}\right)^{T}+\left(\frac{\partial \tilde{\mathbf{X}}^{E}}{\partial \mathbf{x}}\right)\right)=\mathbf{e}^{E}$,

in which only linear terms are retained. Then, since only quadratic terms remain in (13), its implied variation yields linear equations of motion so that the Jacobian (7) of the transformation between Lagrangian and Eulerian frames can be approximated by $J \approx 1$. By this argument, the Eulerian form of VP (13) is

$0=\delta \int_{0}^{T} \iiint_{\Omega_{t}} \rho_{0} \partial_{t} \tilde{\mathbf{X}}^{E} \cdot \mathbf{U}^{E}-\frac{1}{2} \rho_{0}\left|\mathbf{U}^{E}\right|^{2}-\frac{1}{2} \lambda e_{i i}^{E} e_{j j}^{E}-\mu\left(e_{i j}^{E}\right)^{2} \mathrm{~d} x \mathrm{~d} y \mathrm{~d} z \mathrm{~d} t$,

in which the integration is over the moving domain $\Omega_{t}$. The last step is to show that, in the limit of small displacements, the integration can be performed over the fixed domain $\Omega_{0}$ as $\Omega_{t}=\Omega_{0}+\tilde{X}$, meaning that the deformed domain is the reference one subject to deformation. Let us consider a small perturbation of a three-dimensional domain on a length scale that is proportional to $\epsilon$. We can write a general Taylor expansion of the integral in terms of $\epsilon$

$$
\begin{aligned}
& \int_{x_{1}+\epsilon \xi_{1}}^{x_{2}+\epsilon \xi_{2}} \int_{y_{1}+\epsilon \eta_{1}}^{y_{2}+\epsilon \eta_{2}} \int_{z_{1}+\epsilon \zeta_{1}}^{z_{2}+\epsilon \zeta_{2}} f(x, y, z) \mathrm{d} z \mathrm{~d} y \mathrm{~d} x \\
& =\int_{x_{1}}^{x_{2}} \int_{y_{1}}^{y_{2}} \int_{z_{1}}^{z_{2}} f(x, y, z) \mathrm{d} z \mathrm{~d} y \mathrm{~d} x+\epsilon\left(\int_{y_{1}}^{y_{2}} \int_{z_{1}}^{z_{2}} \xi_{2} f\left(x_{2}, y, z\right)-\xi_{1} f\left(x_{1}, y, z\right) \mathrm{d} z \mathrm{~d} y\right. \\
& \left.\quad+\int_{x_{1}}^{x_{2}} \int_{z_{1}}^{z_{2}} \eta_{2} f\left(x, y_{2}, z\right)-\eta_{1} f\left(x, y_{1}, z\right) \mathrm{d} z \mathrm{~d} x+\int_{x_{1}}^{x_{2}} \int_{y_{1}}^{y_{2}} \zeta_{2} f\left(x, y, z_{2}\right)-\zeta_{1} f\left(x, y, z_{1}\right) \mathrm{d} y \mathrm{~d} x\right)+O\left(\epsilon^{2}\right) .
\end{aligned}
$$


The displacement $\tilde{\mathbf{X}}$ can be treated as a small perturbation and linear terms in $\epsilon$ in (18) translate to cubic terms in $\tilde{\mathbf{X}}, \tilde{\mathbf{U}}$ and $\partial_{i} \tilde{X}_{j}$ in (17). Therefore, leaving only quadratic terms and omitting for brevity the $(\cdot)^{E}$ superscript, (17) becomes

$0=\delta \int_{0}^{T} \iiint_{\Omega_{0}} \rho_{0} \partial_{t} \tilde{\mathbf{X}} \cdot \mathbf{U}-\frac{1}{2} \rho_{0}|\mathbf{U}|^{2}-\frac{1}{2} \lambda e_{i i} e_{j j}-\mu e_{i j}^{2} \mathrm{~d} x \mathrm{~d} y \mathrm{~d} z \mathrm{~d} t$

In the limit of small displacement gradients, the following approximations hold:

$\operatorname{tr}(\mathbf{E}) F_{l i}=E_{j j} F_{l i} \approx e_{j j} \delta_{l i}, \quad E_{k i} F_{l k} \approx e_{i k} \delta_{l k}=e_{i l}$.

By either linearizing (10), neglecting the gravity term and using (20) or taking the variation of (13) (or (19)), the classical linearized equations of motion emerge as

$$
\begin{aligned}
\delta \mathbf{U}: & \partial_{t} \tilde{\mathbf{X}}=\mathbf{U}, \\
\delta \tilde{X}_{l}: & \rho_{0} \partial_{t} U_{l}=\partial_{x_{i}}\left(\lambda e_{j j} \delta_{l j}+2 \mu e_{i l}\right) \quad \text { in } \Omega_{0}, \\
\delta \tilde{X}_{l}: & 0=n_{i}\left(\lambda e_{j j} \delta_{l j}+2 \mu e_{i l}\right) \quad \text { on } \quad \partial \Omega_{0} \backslash \partial \Omega_{0}^{b},
\end{aligned}
$$

in which $\Omega_{0}$ denotes the fixed domain after linearization, with associated boundary $\partial \Omega_{0}$ and fixed bottom $\partial \Omega_{0}^{b}$.

\subsection{Coupled model}

The current domain occupied by the fluid is denoted by $\Omega$ and the reference domain occupied by the hyperelastic material by $\Omega_{0}$. For simplicity, we consider a block shape of hyperelastic material. The interface between the fluid and solid domains is parameterized by $\mathbf{X}_{s}=\mathbf{X}\left(L_{s}, b, c, t\right)$ and, at rest, $\mathbf{X}=\mathbf{a}$ for Cartesian $a \in\left[L_{s}, L_{x}\right], b \in$ $\left[0, L_{y}\right], c \in\left[0, L_{z}\right]$, while the fluid domain at rest is $x \in\left[0, L_{s}\right], y \in\left[0, L_{y}\right], z \in\left[0, H_{0}\right]$. The (outward-fromfluid) unit normal at this interface $\mathbf{X}\left(L_{s}, b, c, t\right)$, with $b \in\left[0, L_{y}\right], c \in\left[0, L_{z}\right]$, is $\mathbf{n}=\partial_{b} \mathbf{X} \times \partial_{c} \mathbf{X} /\left|\partial_{b} \mathbf{X} \times \partial_{c} \mathbf{X}\right|$. A schematic diagram of the domain at rest is given in Fig. 3, and hence the above expression is for the outward normal to the fluid domain at the fluid-structure interface.

The moving fluid and elastic domains are defined by

$\Omega: \quad z \in(0, h(x, y, t)), y \in\left(0, L_{y}\right), x \in\left(0, x_{s}(y, z, t)\right) ;$

$\Omega_{0}: \quad a \in\left(L_{s}, L_{x}\right), b \in\left(0, L_{y}\right), c \in\left(0, L_{z}\right)$,

in which $x_{s}$ is a new variable that describes the position of the moving fluid boundary. Since it is at the structure surface, we use a Lagrange multiplier $\gamma=\gamma(b, c, t)$ to equate $x_{s}\left(y=Y\left(L_{s}, b, c, t\right), z=Z\left(L_{s}, b, c, t\right)\right)$ to $X\left(L_{s}, b, c, t\right)$. As the coupled fluid-structure VP, we take the sum of the two VPs, and augment it with the Lagrange-multiplier term as follows:

$$
\begin{aligned}
0= & \delta \int_{0}^{T} \iiint_{\Omega}-\rho\left(\partial_{t} \phi+\frac{1}{2}|\nabla \phi|^{2}+g\left(z-H_{0}\right)\right) \mathrm{d} x \mathrm{~d} y \mathrm{~d} z \\
& +\int_{0}^{L_{y}} \int_{0}^{L_{z}} \rho \gamma\left(x_{s}\left(Y\left(L_{s}, b, c, t\right), Z\left(L_{s}, b, c, t\right), t\right)-X\left(L_{s}, b, c, t\right)\right) \mathrm{d} c \mathrm{~d} b \\
& +\iiint_{\Omega_{0}} \rho_{0} \partial_{t} \mathbf{X} \cdot \mathbf{U}-\frac{1}{2} \rho_{0}|\mathbf{U}|^{2}-\rho_{0} g Z-\frac{1}{2} \lambda[\operatorname{tr}(\mathbf{E})]^{2}-\mu \operatorname{tr}\left(\mathbf{E}^{2}\right) \mathrm{d} a \mathrm{~d} b \mathrm{~d} c \mathrm{~d} t .
\end{aligned}
$$




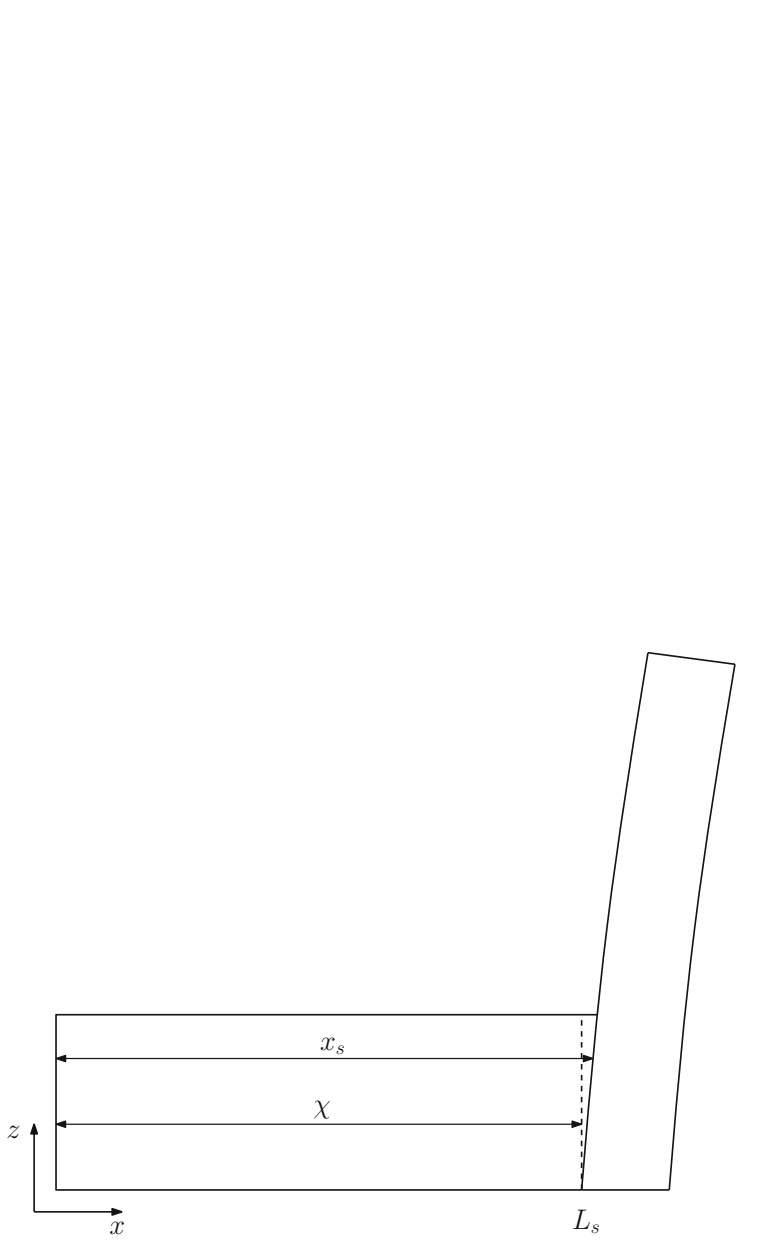

Fig. 4 Definition of the variables used in the VP transformation. Here $x_{s}(y, z, t)$ denotes the position of the fluid-structure interface, and $\chi=L_{s} x / x_{s}(y, z, t)$ denotes the transformation of the domain to one, dimension of which is fixed in the $x$-direction. A cross-section perpendicular to the $y$-direction is shown $\phi, \eta, X, U$

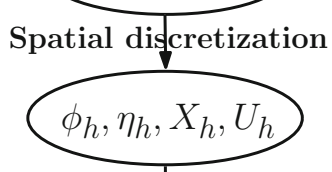

Find X-conjugate momentum $\mathrm{P}$

$$
\phi_{h}, \eta_{h}, X_{h}, P_{h}
$$

Eliminate internal $\phi$

Transform to Hamiltonian form

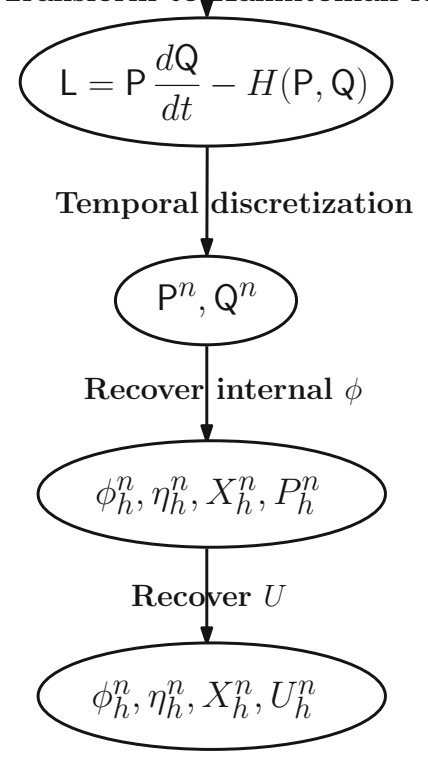

Fig. 5 Flow chart schematically depicting the solution method. The subscript $(\cdot)_{h}$ denotes a spatially discretized function and superscript $(\cdot)^{n}$ the timestep counter

Note that the waterline height $z$ at the fluid-beam interface is implicitly defined by $z=h\left(x_{s}(y, z, t), y, t\right)$, even for the non-breaking waves considered. To avoid the implicit definition, and because it is here easier to work in a fixed domain, we introduce a new horizontal coordinate $\chi=L_{s} x / x_{s}(y, z, t)$ and apply the coordinate transformation $x \rightarrow \chi, y \rightarrow y, z \rightarrow z$ such that the fluid domain $\Omega$ is now redefined as $\chi \in\left(0, L_{s}\right), y \in\left(0, L_{y}\right), z \in$ $(0, h(\chi, y, t))$. Both $x_{s}$ and $\chi$ are indicated in Fig. 4. In this new coordinate system, VP (24) becomes 


$$
\begin{aligned}
0= & \delta \int_{0}^{T} \int_{0}^{L_{s}} \int_{0}^{L_{y}} \int_{0}^{h(\chi, y, t)}-\rho\left(-\frac{\chi}{L_{s}} \partial_{t} x_{s} \partial_{\chi} \phi+\frac{x_{s}}{L_{s}} \partial_{t} \phi\right. \\
& +\frac{1}{2} \frac{L_{s}}{x_{s}}\left(\partial_{\chi} \phi\right)^{2}+\frac{1}{2} \frac{x_{s}}{L_{s}}\left(-\frac{\chi}{x_{s}} \partial_{y} x_{s} \partial_{\chi} \phi+\partial_{y} \phi\right)^{2} \\
& \left.+\frac{1}{2} \frac{x_{s}}{L_{s}}\left(-\frac{\chi}{x_{s}} \partial_{z} x_{s} \partial_{\chi} \phi+\partial_{z} \phi\right)^{2}+g\left(z-H_{0}\right) \frac{x_{s}}{L_{s}}\right) \mathrm{d} z \mathrm{~d} y \mathrm{~d} \chi \\
& +\int_{0}^{L_{y}} \int_{0}^{L_{z}} \rho \gamma\left(x_{s}\left(Y\left(L_{s}, b, c, t\right), Z\left(L_{s}, b, c, t\right), t\right)-X\left(L_{s}, b, c, t\right)\right) \mathrm{d} c \mathrm{~d} b \\
& +\int_{L_{s}}^{L_{x}} \int_{0}^{L_{y}} \int_{0}^{L_{z}} \rho_{0} \partial_{t} \mathbf{X} \cdot \mathbf{U}-\frac{1}{2} \rho_{0}|\mathbf{U}|^{2}-\rho_{0} g Z-\frac{1}{2} \lambda[\operatorname{tr}(\mathbf{E})]^{2}-\mu \operatorname{tr}\left(\mathbf{E}^{2}\right) \mathrm{d} c \mathrm{~d} b \mathrm{~d} a \mathrm{~d} t .
\end{aligned}
$$

\subsection{Linearized wave-beam dynamics for FSI}

We linearize (25) around a state of rest. Small-amplitude perturbations around this rest state are introduced as follows:

$x_{s}=L_{s}+\tilde{x}_{s}, \quad \phi=0+\phi, \quad h=H_{0}+\eta, \quad \mathbf{X}=\mathbf{x}+\tilde{\mathbf{X}}, \quad \mathbf{U}=\mathbf{0}+\mathbf{U}, \quad \gamma=0+\gamma$.

After some manipulations (described in detail in "Appendix A"), one arrives at the following linearized VP:

$$
\begin{aligned}
0= & \delta \int_{0}^{T} \int_{0}^{L_{s}} \int_{0}^{L_{y}} \rho \partial_{t} \eta \phi_{f}-\frac{1}{2} \rho g \eta^{2}-\int_{0}^{H_{0}} \frac{1}{2} \rho|\nabla \phi|^{2} \mathrm{~d} z \mathrm{~d} y \mathrm{~d} x \\
& +\int_{0}^{L_{y}} \int_{0}^{H_{0}} \rho \partial_{t} \tilde{X}_{s} \phi_{s} \mathrm{~d} z \mathrm{~d} y \\
& +\int_{L_{s}}^{L_{x}} \int_{0}^{L_{y}} \int_{0}^{L_{z}} \rho_{0} \partial_{t} \tilde{\mathbf{X}} \cdot \mathbf{U}-\frac{1}{2} \rho_{0}|\mathbf{U}|^{2}-\frac{1}{2} \lambda e_{i i} e_{j j}-\mu e_{i j}^{2} \mathrm{~d} z \mathrm{~d} y \mathrm{~d} x \mathrm{~d} t .
\end{aligned}
$$

We used the definitions of the velocity potentials $\phi_{s}=\phi\left(L_{s}, y, z, t\right)$ and $\phi_{f}=\phi(x, y, h(x, y, t), t)$, at the beam interface and the free surface, respectively. The coupling term (27b), derived here, is equivalent to the ad hoc one proposed in [11]. After using the temporal endpoint conditions $\delta \tilde{\mathbf{X}}(\mathbf{x}, 0)=\delta \tilde{\mathbf{X}}(\mathbf{x}, T)=0$ and $\delta \eta(x, y, 0)=\delta \eta(x, y, T)=0$, the variation in (27) yields

$\delta \phi_{s}: \quad \partial_{t} \tilde{X}_{s}=\partial_{x} \phi \quad$ at $x=L_{s}$,

$\delta \tilde{X}_{j}\left(L_{s}, y, z, t\right): \quad-\delta_{1 j} \rho \partial_{t} \phi_{s}=T_{1 j} \quad$ at $\quad x=L_{s}$,

$\delta \phi_{f}: \quad \partial_{t} \eta=\partial_{z} \phi \quad$ at $z=H_{0}$

$\delta \eta: \partial_{t} \phi_{f}=-g \eta \quad$ at $z=H_{0}$,

$\delta \phi: \nabla^{2} \phi=0$ in $\Omega$,

$\delta \mathbf{U}: \quad \partial_{t} \tilde{\mathbf{X}}=\mathbf{U}$ in $\Omega_{0}$,

$\delta \tilde{X}_{j}: \quad \rho_{0} \partial_{t} \mathbf{U}_{j}=\nabla_{k} T_{j k}$ in $\Omega_{0}$

with $\Omega_{0}: x \in\left[L_{s}, L_{x}\right], y \in\left[0, L_{y}\right], z \in\left[0, L_{z}\right], \quad \Omega: \chi \in\left[0, L_{s}\right], y \in\left[0, L_{y}\right], z \in\left[0, H_{0}\right]$ and linear stress tensor $T_{i j}=\lambda \delta_{i j} e_{k k}+2 \mu e_{i j}$.

To further simplify computations, we introduce non-dimensional variables. We choose a length scale $D$, e.g., beam length; thereafter other units are nondimensionalized using 
$V=\sqrt{g D}, \quad T=\frac{D}{V}, \quad M=\rho D^{3}$.

Then, we transform coordinates and variables to non-dimensional ones using

$$
\begin{aligned}
& x \rightarrow D x \quad y \rightarrow D y \quad z \rightarrow D z \quad \eta \rightarrow D \eta, \\
& \rho \rightarrow \frac{M}{D^{3}} \rho \quad \rho_{0} \rightarrow \frac{M}{D^{3}} \rho_{0} \quad \phi \rightarrow V D \phi, \\
& \mathbf{X} \rightarrow D \mathbf{X} \quad \lambda \rightarrow \frac{M}{D T^{2}} \lambda \quad \mu \rightarrow \frac{M}{D T^{2}} \mu .
\end{aligned}
$$

Using (30) enables transformation of the whole Lagrangian to the non-dimensional one $\mathcal{L} \rightarrow M V^{2} \mathcal{L}$, whence the final simplified Lagrangian from the VP (27) becomes

$$
\begin{aligned}
\mathcal{L}= & \int_{0}^{L_{s}} \int_{0}^{L_{y}}\left[\partial_{t} \eta \phi_{f}-\frac{1}{2} \eta^{2}-\int_{0}^{H_{0}} \frac{1}{2}|\nabla \phi|^{2} \mathrm{~d} z\right] \mathrm{d} y \mathrm{~d} x+\int_{0}^{L_{y}} \int_{0}^{H_{0}} \partial_{t} X_{s} \phi_{s} \mathrm{~d} z \mathrm{~d} y \\
& +\int_{L_{s}}^{L_{x}} \int_{0}^{L_{y}} \int_{0}^{L_{z}} \rho_{0} \partial_{t} \mathbf{X} \cdot \mathbf{U}-\frac{1}{2} \rho_{0}|\mathbf{U}|^{2}-\frac{1}{2} \lambda e_{i i} e_{j j}-\mu e_{i j}^{2} \mathrm{~d} z \mathrm{~d} y \mathrm{~d} x
\end{aligned}
$$

with, we recall, $e_{i j}=\frac{1}{2}\left(\partial_{i} X_{j}+\partial_{j} X_{i}\right)$. Hereafter, although the tilde over the $\mathbf{X}$ has been dropped for simplicity of notation, it still denotes the displacement rather than the actual beam position.

\section{Solution method for the linear system}

In Fig. 5, we portray the discretization procedure of the VP with Lagrangian (31). We reduce the system to Hamiltonian form, in which a known stable time discretization scheme can be applied. Though ultimately we seek a space-time discrete system of equations, it is much easier to work with the space-discretized system than with the continuous one, as it invites the use of matrix inverses and partial derivatives instead of functional ones. Therefore, we first proceed with spatial discretization by using continuous $C^{0}$-Galerkin finite-element expansions directly substituted into the VP. Since the variable $\mathbf{X}$ is conjugated through coupling to both $\mathbf{U}$ and $\phi$, the first step is to find its single conjugate momentum $\mathbf{P}$. It transpires that the interior $\phi$ degrees of freedom are not independent, and can be expressed in terms of the free-surface ones $\phi_{f}$ and $\mathbf{P}$ at the common boundary. The resulting system has a standard Hamiltonian structure with Lagrangian $\mathrm{L}=\mathrm{PdQ} / \mathrm{d} t-\mathrm{H}(\mathrm{P}, \mathrm{Q}, t)$, where $\mathrm{Q}=\mathrm{Q}(t)$ and $\mathrm{P}=\mathrm{P}(t)$ are the conjugate vectors of unknowns, see Fig. 5. For such a system, stable, second-order, conservative temporal schemes such as the Störmer-Verlet method are known. One is thus left with a fully discretized VP and the resulting algebraic equations of motion follow. To avoid computing full-system matrix inverses, we reintroduce $\phi$ in the interior, together with $\mathbf{U}$ instead of $\mathbf{P}$ at properly determined time levels. Details are provided next.

\subsection{FEM space discretization}

To find a spatial discretization, $C^{0}$-Galerkin finite-element expansions of the variables are, given an appropriate mesh tessellation of the fixed fluid and beam domains, substituted directly into the VP. The basis functions are $\tilde{\varphi}_{i}(x, y, z)$ in the fluid domain with the limiting basis function $\tilde{\varphi}_{\alpha}(x, y)=\tilde{\varphi}_{\alpha}\left(x, y, z=H_{0}\right)$ at the free surface $z=H_{0}$, and $\tilde{X}_{k}(x, y, z)$ in the structural domain. Both the fixed fluid and beam domains have coordinates $\mathbf{x}=(x, y, z)=\left(x_{1}, x_{2}, x_{3}\right)$. At the common interface $x=L_{s}$ (see Fig. 3), we assume that the respective meshes join up with common nodes. However, since there are two meshes, these nodes are denoted by indices $m$ and $n$ on the fluid mesh and by $\tilde{m}$ and $\tilde{n}$ on the solid mesh. There is a mapping between these two node sets, namely 
$m=m(\tilde{m})$. Here, $i$ and $j$ denote nodes in the fluid domain, $\alpha$ and $\beta$ nodes at its surface, $m$ and $n$ or $\tilde{m}$ and $\tilde{n}$ nodes at the common fluid-structure boundary, and $k$ and $l$ nodes in the structure domain. Primed indices refer to the nodes below the water surface, and $\alpha_{n}$ denotes the surface nodes at the common boundary. Indices $a, b=1,2,3$ are the coordinate indices used for $\mathbf{X}$ and $\mathbf{x}$. The Einstein summation convention is assumed for all indices. Finally, with the subscript $h$ denoting the numerical approximations, the expansions are

$$
\begin{aligned}
& \phi_{h}(\mathbf{x}, t)=\phi_{i}(t) \tilde{\varphi}_{i}(\mathbf{x}), \quad \phi_{f h}(x, y, t)=\phi_{\alpha}(t) \tilde{\varphi}_{\alpha}(x, y), \quad \eta_{h}(x, y, t)=\eta_{\alpha}(t) \tilde{\varphi}_{\alpha}(x, y), \\
& X_{h}^{a}(\mathbf{x}, t)=X_{k}^{a}(t) \tilde{X}_{k}(\mathbf{x}), \quad U_{h}^{a}(\mathbf{x}, t)=U_{k}^{a}(t) \tilde{X}_{k}(\mathbf{x}) .
\end{aligned}
$$

Substitution of (32) into (31) yields the spatially discrete Lagrangian function

$L=\dot{\eta}_{\alpha} M_{\alpha \beta} \phi_{\beta}+\dot{X}_{k}^{a} N_{k l} U_{l}^{a}+\dot{X}_{\widetilde{m}}^{1} W_{\widetilde{m} n} \phi_{n}-H(\eta, \phi, X, U)$,

with Hamiltonian

$$
H(\eta, \phi, X, U)=\frac{1}{2} \eta_{\alpha} M_{\alpha \beta} \eta_{\beta}+\frac{1}{2} \phi_{i} A_{i j} \phi_{j}+\frac{1}{2} U_{k}^{a} N_{k l} U_{l}^{a}-\frac{1}{2} X_{k}^{a} E_{k l}^{a b} X_{l}^{b},
$$

wherein a superscript dot indicates a time derivative, and in which the matrices are given by

$$
\begin{aligned}
& M_{\alpha \beta}=\int_{x} \int_{y} \tilde{\varphi}_{\alpha} \tilde{\varphi}_{\beta} \mathrm{d} y \mathrm{~d} x, \quad A_{i j}=\int_{\Omega} \nabla \tilde{\varphi}_{i} \cdot \nabla \tilde{\varphi}_{j} \mathrm{~d} V \\
& W_{\widetilde{m} n}=\int_{y} \int_{0}^{H_{0}} \tilde{X}_{\tilde{m}} \tilde{\varphi}_{n} \mathrm{~d} z \mathrm{~d} y, \quad N_{k l}=\rho_{0} \int_{\Omega_{0}} \tilde{X}_{k} \tilde{X}_{l} \mathrm{~d} V \\
& B_{k l}^{a b}=\int_{\Omega_{0}} \frac{\partial \tilde{X}_{k}}{\partial x_{a}} \frac{\partial \tilde{X}_{l}}{\partial x_{b}} \mathrm{~d} V, \quad E_{k l}^{a b}=\lambda B_{k l}^{a b}+\mu\left(B_{k l}^{c c} \delta_{a b}+B_{k l}^{b a}\right) .
\end{aligned}
$$

Given that in both fluid and beam domains the basis functions come from the same function space, we can identify $\tilde{X}_{\widetilde{m}} \equiv \tilde{\phi}_{m(\widetilde{m})}$. In other words, if the numbering is taken into account, at the fluid-beam inferface, basis functions are the same in both the fluid and the beam. The matrices in (35) are symmetric; in particular, we highlight that

$$
B_{k l}^{a b}=B_{l k}^{b a} \quad \text { and } \quad E_{k l}^{a b}=E_{l k}^{b a}
$$

Unlike in the continuous case, $c f$. remarks after (4), the Dirichlet boundary condition can be incorporated directly into the Lagrangian, i.e., by imposing $X_{k_{b}}^{a}=0$ and $U_{k_{b}}^{a}=0$, with $(\cdot)_{k_{b}}$ denoting the structure-base nodes. Then (33) becomes

$$
\begin{aligned}
& L=\dot{\eta}_{\alpha} M_{\alpha \beta} \phi_{\beta}+\dot{X}_{k^{\prime}}^{a} N_{k^{\prime} l^{\prime}} U_{l^{\prime}}^{a}+\dot{X}_{\widetilde{m}^{\prime}}^{1} W_{\widetilde{m}^{\prime} n} \phi_{n}-H(\eta, \phi, X, U), \\
& H(\eta, \phi, X, U)=\frac{1}{2} \eta_{\alpha} M_{\alpha \beta} \eta_{\beta}+\frac{1}{2} \phi_{i} A_{i j} \phi_{j}+\frac{1}{2} U_{k^{\prime}}^{a} N_{k^{\prime} l^{\prime}} U_{l^{\prime}}^{a}-\frac{1}{2} X_{k^{\prime}}^{a} E_{k^{\prime} l^{\prime}}^{a b} X_{l^{\prime}}^{b},
\end{aligned}
$$

with primed structural indices denoting nodes excluding those at the beam bottom. The next step is to compute the momentum conjugate to $X_{k^{\prime}}^{a}$,

$R_{k^{\prime}}^{a}=\frac{\partial L}{\partial \dot{X}_{k^{\prime}}^{a}}=N_{k^{\prime} l^{\prime}} U_{l^{\prime}}^{a}+\delta_{a 1} \delta_{k^{\prime} \tilde{m}^{\prime}} W_{\widetilde{m}^{\prime} n} \phi_{n}$,

in which $\delta$ is the Kronecker delta symbol. Rearrangement of (38) yields 
$U_{k^{\prime}}^{a}=N_{k^{\prime} l^{\prime}}^{-1} R_{l^{\prime}}^{a}-\delta_{a 1} N_{k^{\prime} l^{\prime}}^{-1} \delta_{l^{\prime} \widetilde{m}^{\prime}} W_{\widetilde{m}^{\prime} n} \phi_{n}$,

in which it is to be noted that $N_{k^{\prime} l^{\prime}}^{-1}$ is the inverse not of the full matrix $N_{k l}$, but of the system excluding the nodes at the beam bottom. Therefore, after using $R_{k^{\prime}}^{a}$ instead of $U_{k^{\prime}}^{a}$, the Lagrangian takes the form

$L=\dot{\eta}_{\alpha} M_{\alpha \beta} \phi_{\beta}+\dot{X}_{k^{\prime}}^{a} R_{k^{\prime}}^{a}-H\left(\phi_{\alpha}, \eta_{\alpha}, X_{k^{\prime}}^{a}, R_{k^{\prime}}^{a}\right)$,

in which the Hamiltonian (computed using the Lagrangian $L$ in (33) and (39)) is given by

$$
\begin{aligned}
H\left(\phi_{\alpha}, \eta_{\alpha}, X_{k^{\prime}}^{a}, R_{k^{\prime}}^{a}\right)= & \dot{\eta}_{\alpha} M_{\alpha \beta} \phi_{\beta}+\dot{X}_{k^{\prime}}^{a} R_{k^{\prime}}^{a}-L=\frac{1}{2} \eta_{\alpha} M_{\alpha \beta} \eta_{\beta}+\frac{1}{2} \phi_{i} A_{i j} \phi_{j}+\frac{1}{2} \phi_{m} \widetilde{M}_{m n} \phi_{n} \\
& -R_{k^{\prime}}^{1} N_{k^{\prime} l^{\prime}}^{-1} \delta_{l^{\prime} \widetilde{m}^{\prime}} W_{\widetilde{m}^{\prime} n} \phi_{n}+\frac{1}{2} R_{k^{\prime}}^{a} N_{k^{\prime} l^{\prime}}^{-1} R_{l^{\prime}}^{a}+\frac{1}{2} X_{k^{\prime}}^{a} E_{k^{\prime} l^{\prime}}^{a b} X_{l^{\prime}}^{b},
\end{aligned}
$$

in which

$\widetilde{M}_{m n}=\left(N^{-1}\right)_{\widetilde{m}^{\prime} \tilde{n}^{\prime}} W_{\widetilde{m}^{\prime} m} W_{\tilde{n}^{\prime} n}$.

To facilitate the computations, we introduce the vector $P$ defined by

$R_{k^{\prime}}^{a}=N_{k^{\prime} l^{\prime}} P_{l^{\prime}}^{a}$,

which obviates the need to compute the inverse of the full matrix $N$, instead requiring only the part in the definition of $\widetilde{M}_{m n}$. The inverse $\left(N^{-1}\right)_{\widetilde{m}^{\prime} \tilde{n}^{\prime}}$ in (42) is the submatrix of the inverse of $N_{k^{\prime} l^{\prime}}$ including interface but excluding beam-bottom nodes. Therefore, the substitution of (43) into (40) using (41) yields

$L=\dot{\eta}_{\alpha} M_{\alpha \beta} \phi_{\beta}+\dot{X}_{k^{\prime}}^{a} N_{k^{\prime} l^{\prime}} P_{l^{\prime}}^{a}-H\left(\phi_{\alpha}, \eta_{\alpha}, X_{k^{\prime}}^{a}, P_{k^{\prime}}^{a}\right)$

with the Hamiltonian

$$
\begin{aligned}
H\left(\phi_{\alpha}, \eta_{\alpha}, X_{k^{\prime}}^{a}, P_{k^{\prime}}^{a}\right)= & \frac{1}{2} \eta_{\alpha} M_{\alpha \beta} \eta_{\beta}+\frac{1}{2} \phi_{i} A_{i j} \phi_{j}+\frac{1}{2} \phi_{m} \widetilde{M}_{m n} \phi_{n} \\
& -P_{\widetilde{m}^{\prime}}^{1} W_{\widetilde{m}^{\prime} n} \phi_{n}+\frac{1}{2} P_{k^{\prime}}^{a} N_{k^{\prime} l^{\prime}} P_{l^{\prime}}^{a}+\frac{1}{2} X_{k^{\prime}}^{a} E_{k^{\prime} l^{\prime}}^{a b} X_{l^{\prime}}^{b}
\end{aligned}
$$

The fact that not all terms in (45) are positive definite will be discussed in more detail later. Note that the Hamiltonian depends explicitly on only the surface degrees of freedom $\phi_{\alpha}$. Therefore, we are able to eliminate the interior degrees of freedom $\phi_{i^{\prime}}$, with the primed index $i^{\prime}$ denoting the nodes in the interior of the fluid excluding those on the free surface, in order to reduce the system to the general Hamiltonian form. Therefore, we derive the equations of motion by applying the VP to the Lagrangian (44); after rearranging and using arbitrariness of respective variations as well as suitable end-point conditions, we obtain

$$
\begin{aligned}
0= & \int_{0}^{t_{1}} L \mathrm{~d} t \\
=\int_{0}^{t_{1}}\{ & \dot{\eta}_{\alpha} M_{\alpha \beta} \delta \phi_{\beta}-M_{\alpha \beta} \dot{\phi}_{\beta} \delta \eta_{\alpha}-\eta_{\alpha} M_{\alpha \beta} \delta \eta_{\beta}-\phi_{i} A_{i j} \delta \phi_{j}-\phi_{m} \widetilde{M}_{m n} \delta \phi_{n} \\
& +\left(W_{\widetilde{m}^{\prime} n} \phi_{n} \delta P_{\widetilde{m}^{\prime}}^{1}+P_{\widetilde{m}^{\prime}}^{1} W_{\widetilde{m}^{\prime} n} \delta \phi_{n}\right) \\
& \left.+\left(\dot{X}_{k^{\prime}}^{a} N_{k^{\prime} l^{\prime}} \delta P_{l^{\prime}}^{a}-N_{k^{\prime} l^{\prime}} \dot{P}_{l^{\prime}}^{a} \delta X_{k^{\prime}}^{a}-P_{k^{\prime}}^{a} N_{k^{\prime} l^{\prime}} \delta P_{l^{\prime}}^{a}\right)-X_{k^{\prime}}^{a} E_{k^{\prime} l^{\prime}}^{a b} \delta X_{l^{\prime}}^{b}\right\} \mathrm{d} t .
\end{aligned}
$$


Hence, by renaming certain indices, the following equations are obtained

$$
\begin{aligned}
& \delta \eta_{\beta}: \quad \dot{\phi}_{\alpha}=-\eta_{\alpha}, \\
& \delta \phi_{\alpha}: \quad M_{\alpha \beta} \dot{\eta}_{\beta}=\phi_{i} A_{i \alpha}+\left(\phi_{m} \widetilde{M}_{m n}-P_{\widetilde{m}^{\prime}}^{1} W_{\widetilde{m}^{\prime} n}\right) \delta_{\alpha n}, \\
& \delta \phi_{j^{\prime}}: \quad \phi_{i} A_{i j^{\prime}}=\left(-\phi_{m} \widetilde{M}_{m n}+P_{\widetilde{m}^{\prime}}^{1} W_{\widetilde{m}^{\prime} n}\right) \delta_{n j^{\prime}}, \\
& \delta P_{k^{\prime}}^{a}: \quad N_{k^{\prime} l^{\prime}} \dot{X}_{l^{\prime}}^{a}=N_{k^{\prime} l^{\prime}} P_{l^{\prime}}^{a}-\delta_{a 1} \delta_{k^{\prime} \tilde{m}^{\prime}} W_{\widetilde{m}^{\prime} n} \phi_{n}, \\
& \delta X_{k^{\prime}}^{a}: \quad N_{k^{\prime} l^{\prime}} \dot{P}_{l^{\prime}}^{a}=-E_{k^{\prime} l^{\prime}}^{a b} X_{l^{\prime}}^{b},
\end{aligned}
$$

in which the new coupling terms introduced by the present formulation are underlined. If we define the matrix

$C_{i^{\prime} j^{\prime}}=A_{i^{\prime} j^{\prime}}+\delta_{i^{\prime} m} \tilde{M}_{m n} \delta_{n j^{\prime}}$

(47c) can be split into internal and surface degrees of freedom and inverted to express internal ones in terms of surface ones and $P$ at the interface

$\phi_{i^{\prime}}=C_{i^{\prime} j^{\prime}}^{-1}\left(-\phi_{\alpha} A_{\alpha j^{\prime}}+P_{\widetilde{m}^{\prime}}^{1} W_{\widetilde{m}^{\prime} n} \delta_{n j^{\prime}}-\phi_{\alpha} \delta_{\alpha m} \widetilde{M}_{m n} \delta_{n j^{\prime}}\right)$.

The interior degrees of freedom are removed from the Lagrangian by substituting (49) into (40) to obtain

$$
L=\dot{\eta}_{\alpha} M_{\alpha \beta} \phi_{\beta}-\frac{1}{2} \eta_{\alpha} M_{\alpha \beta} \eta_{\beta}-\frac{1}{2} \phi_{\alpha} D_{\alpha \beta} \phi_{\beta}+P_{k^{\prime}}^{a} G_{k^{\prime} \alpha}^{a} \phi_{\alpha}+P_{k^{\prime}}^{a} N_{k^{\prime} l^{\prime}} \dot{X}_{l^{\prime}}^{a}-\frac{1}{2} P_{k^{\prime}}^{a} F_{k^{\prime} l^{\prime}}^{a b} P_{l^{\prime}}^{b}-\frac{1}{2} X_{k^{\prime}}^{a} E_{k^{\prime} l^{\prime}}^{a b} X_{l^{\prime}}^{b}
$$

where Schur decomposition matrices $B, F$ and $G$ have been introduced; their explicit forms are omitted. The structure of (50) is as follows: the first line describes the fluid, the second the coupling, and the third the beam. In a more visual matrix notation, (50) has the structure

$$
L=(\dot{\eta}, \dot{\mathbf{X}})\left(\begin{array}{c}
M \phi \\
N
\end{array}\right)-\frac{1}{2}(\eta, \mathbf{X})\left(\begin{array}{cc}
M & 0 \\
0 & E
\end{array}\right)\left(\begin{array}{l}
\eta \\
\mathbf{X}
\end{array}\right)-\frac{1}{2}(\phi, \mathbf{P})\left(\begin{array}{cc}
D & -G^{T} \\
-G & F
\end{array}\right)\left(\begin{array}{l}
\phi \\
\mathbf{P}
\end{array}\right)
$$

The classical Hamilton's equations of an abstract system emerge when we introduce a generalized coordinate vector and its conjugate vector, i.e.

$$
\begin{aligned}
& \mathrm{Q}=\left(\eta_{1}, \ldots, \eta_{N_{f}}, X_{1}^{1}, \ldots, X_{N_{b}}^{1}, X_{1}^{2}, \ldots, X_{N_{b}}^{2}, X_{1}^{3}, \ldots, X_{N_{b}}^{3}\right), \\
& \mathrm{P}=\left(M_{1 \alpha} \phi_{\alpha}, \ldots, M_{N_{f} \alpha} \phi_{\alpha}, N_{1 k^{\prime}} P_{k^{\prime}}^{1}, \ldots, N_{N_{b} k^{\prime}} P_{k^{\prime}}^{1}, N_{1 k^{\prime}} P_{k^{\prime}}^{2}, \ldots, N_{N_{b} k^{\prime}} P_{k^{\prime}}^{2}, N_{1 k^{\prime}} P_{k^{\prime}}^{3}, \ldots, N_{N_{b} k^{\prime}} P_{k^{\prime}}^{3}\right),
\end{aligned}
$$

with $N_{f}$ degrees of freedom at the free surface and $N_{b}$ degrees of freedom in the beam (recall, fixed-bottom nodes are excluded), using which the Lagrangian can be written in the form:

$\mathrm{L}=\frac{\mathrm{dQ}}{\mathrm{d} t} \cdot \mathrm{P}-\mathrm{H}(\mathrm{Q}, \mathrm{P})$

with Hamiltonian $\mathrm{H}(\mathrm{P}, \mathrm{Q})$. After introducing the following (symmetric) matrices

$$
\begin{aligned}
\mathrm{M}_{\mathrm{Q}} & =\left(\begin{array}{cc}
M & 0 \\
0 & E
\end{array}\right), \\
\mathrm{M}_{\mathrm{P}} & =\left(\begin{array}{cc}
M^{-1} D M^{-1} & -M^{-1} G^{T} N^{-1} \\
-N^{-1} G M^{-1} & N^{-1} F N^{-1}
\end{array}\right),
\end{aligned}
$$


we can write the Hamiltonian in (53) as

$H(Q, P)=\frac{1}{2} Q^{\top} M_{Q} Q+\frac{1}{2} P^{\top} M_{P} P$

\subsection{Time discretization}

The Störmer-Verlet scheme (see [14] for a definition, and [15] for a variational derivation) is used to discretize (53) to second-order accuracy in time. The resulting difference equations are

$$
\begin{aligned}
& \mathrm{P}^{n+1 / 2}=\mathrm{P}^{n}-\frac{1}{2} \Delta t \frac{\partial \mathrm{H}\left(\mathrm{Q}^{n}, \mathrm{P}^{n+1 / 2}\right)}{\partial \mathrm{Q}^{n}}, \\
& \mathrm{Q}^{n+1}=\mathrm{Q}^{n}+\frac{1}{2} \Delta t\left(\frac{\partial \mathrm{H}\left(\mathrm{Q}^{n}, \mathrm{P}^{n+1 / 2}\right)}{\partial \mathrm{P}^{n+1 / 2}}+\frac{\partial \mathrm{H}\left(\mathrm{Q}^{n+1}, \mathrm{P}^{n+1 / 2}\right)}{\partial \mathrm{P}^{n+1 / 2}}\right), \\
& \mathrm{P}^{n+1}=\mathrm{P}^{n+1 / 2}-\frac{1}{2} \Delta t \frac{\partial \mathrm{H}\left(\mathrm{Q}^{n+1}, \mathrm{P}^{n+1 / 2}\right)}{\partial \mathrm{Q}^{n+1}} .
\end{aligned}
$$

In the linear case considered, for which the Hamiltonian is given by (55), (56) yields the explicit scheme

$$
\begin{aligned}
& \mathrm{P}^{n+1 / 2}=\mathrm{P}^{n}-\frac{1}{2} \Delta t \mathrm{M}_{\mathrm{Q}} \mathrm{Q}^{n} \\
& \mathrm{Q}^{n+1}=\mathrm{Q}^{n}+\Delta t \mathrm{M}_{\mathrm{P}} \mathrm{P}^{n+1 / 2}, \\
& \mathrm{P}^{n+1}=\mathrm{P}^{n+1 / 2}-\frac{1}{2} \Delta t \mathrm{M}_{\mathrm{Q}} \mathrm{Q}^{n+1}
\end{aligned}
$$

After some manipulations (described in detail in "Appendix B"), in terms of original physical variables, the discretization to be implemented is

$$
\begin{aligned}
& \phi_{\alpha}^{n+1 / 2}=\phi_{\alpha}^{n}-\frac{1}{2} \Delta t \eta_{\alpha}^{n},
\end{aligned}
$$

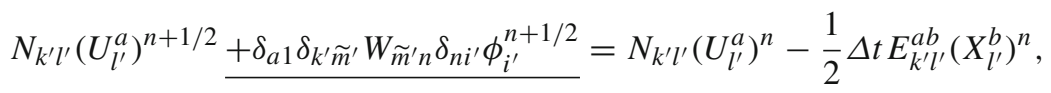

$$
\begin{aligned}
& +\delta_{a 1} \delta_{k^{\prime} \widetilde{m}^{\prime}} W_{\widetilde{m}^{\prime} n} \phi_{n}^{n}-\delta_{a 1} \delta_{k^{\prime} \widetilde{m}^{\prime}} W_{\widetilde{m}^{\prime} n} \delta_{n \alpha} \phi_{\alpha}^{n+1 / 2}, \\
& A_{i^{\prime} j^{\prime}} \phi_{i^{\prime}}^{n+1 / 2}-\left(U_{\widetilde{m}^{\prime}}^{1}\right)^{n+1 / 2} W_{\widetilde{m}^{\prime} n} \delta_{n j^{\prime}}=-A_{\alpha j^{\prime}} \phi_{\alpha}^{n+1 / 2}, \\
& M_{\alpha \beta} \eta_{\beta}^{n+1}=M_{\alpha \beta} \eta_{\beta}^{n}+\Delta t A_{\alpha i} \phi_{i}^{n+1 / 2}-\Delta t\left(U_{\widetilde{m}^{\prime}}^{1}\right)^{n+1 / 2} W_{\widetilde{m}^{\prime} n} \delta_{n \alpha}, \\
& \left(X_{k^{\prime}}^{a}\right)^{n+1}=\left(X_{k^{\prime}}^{a}\right)^{n}+\Delta t\left(U_{k^{\prime}}^{a}\right)^{n+1 / 2}, \\
& \phi_{\alpha}^{n+1}=\phi_{\alpha}^{n+1 / 2}-\frac{1}{2} \Delta t \eta_{\alpha}^{n+1} \text {, } \\
& N_{k^{\prime} l^{\prime}}\left(U_{l^{\prime}}^{a}\right)^{n+1} \underline{+\delta_{a 1} \delta_{k^{\prime} \tilde{m}^{\prime}} W_{\widetilde{m}^{\prime} n} \delta_{n i^{\prime}} \phi_{i^{\prime}}^{n+1}}=N_{k^{\prime} l^{\prime}}\left(U_{l^{\prime}}^{a}\right)^{n+1 / 2}-\frac{1}{2} \Delta t E_{k^{\prime} l^{\prime}}^{a b}\left(X_{l^{\prime}}^{b}\right)^{n+1} \\
& \underline{+\delta_{a 1} \delta_{k^{\prime} \tilde{m}^{\prime}} W_{\widetilde{m}^{\prime} n} \phi_{n}^{n+1 / 2}-\delta_{a 1} \delta_{k^{\prime} \widetilde{m}^{\prime}} W_{\widetilde{m}^{\prime} n} \delta_{n \alpha} \phi_{\alpha}^{n+1}}
\end{aligned}
$$

We remark that Eqs. (58a), (58d), (58e) and (58f) can be solved in the separate fluid and structure domains, while (58b), (58c), (58g) and (58h) have to be solved in both domains simultaneously. Therefore, the scheme is a variant of the mixed partitioned-monolithic approach, see e.g., [16]. 
Table 1 Parameter values used in the $2 \mathrm{D}$ computations

\begin{tabular}{lll}
\hline Parameter & Value & Comment \\
\hline$g$ & $9.8 \mathrm{~m} / \mathrm{s}^{2}$ & Gravitational acceleration \\
$L_{x} \times H_{0}$ & $20 \mathrm{~m} \times 10 \mathrm{~m}$ & Water domain \\
$L_{x}^{B} \times L_{z}^{B}$ & $2 \mathrm{~m} \times 20 \mathrm{~m}$ & Beam domain \\
$\rho$ & $1000 \mathrm{~kg} / \mathrm{m}^{2}$ & Water density \\
$\rho_{0}$ & $7700 \mathrm{~kg} / \mathrm{m}^{2}$ & Beam density (steel) \\
$\lambda$ & $1 \times 10^{7} \mathrm{~N} / \mathrm{m}$ & First Lamé constant \\
$\mu$ & $1 \times 10^{7} \mathrm{~N} / \mathrm{m}$ & Second Lamé constant \\
$N_{x}^{\mathrm{W}} \times N_{z}^{W}$ & $20 \times 10$ & No. of elements in water \\
$N_{x}^{\mathrm{B}} \times N_{z}^{B}$ & $4 \times 20$ & No. of elements in beam \\
\hline
\end{tabular}

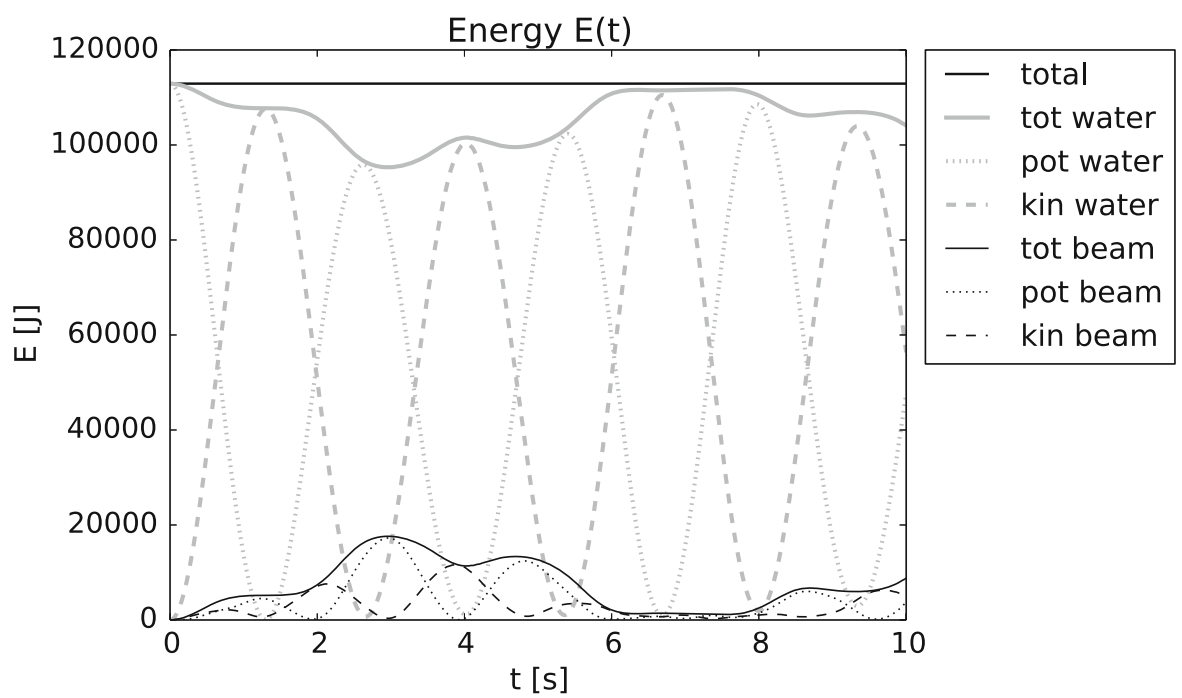

Fig. 6 Energy apportionment (in J) in the 2D system. From top to bottom (see key), curves represent energies of the total system (medium, horizontal), total water (thick, wavy), potential/kinetic water (thick dotted/dashed oscillatory), total beam (thin, wavy) and potential/kinetic beam (thin dotted/dashed oscillatory)

The Firedrake software (see start of Sect.4) used to obtain 3D results accepts equations in the weak form as an input. Therefore, the weak-form equivalent of (58), with more general structural geometry, is

$$
\begin{aligned}
& \int v \phi^{n+1 / 2} \mathrm{~d} S_{f}=\int v\left(\phi^{n}-\frac{1}{2} \Delta t \eta^{n}\right) \mathrm{d} S_{f} \\
& \int \rho_{0} \mathbf{v} \cdot \mathbf{U}^{n+1 / 2} \mathrm{~d} V_{S}+\int \mathbf{n} \cdot \mathbf{v} \phi^{n+1 / 2} \mathrm{~d} S_{S} \\
& =\rho_{0} \int \mathbf{v} \cdot \mathbf{U}^{n} \mathrm{~d} V_{S}-\frac{1}{2} \Delta t \int\left(\lambda \nabla \cdot \mathbf{v} \nabla \cdot \mathbf{X}^{n}+\mu \partial_{a} X_{b}^{n}\left(\partial_{a} v_{b}+\partial_{b} v_{a}\right)\right) \mathrm{d} V_{S}+\int \mathbf{n} \cdot \mathbf{v} \phi^{n} \mathrm{~d} S_{S}, \\
& \int \nabla v \cdot \nabla \phi^{n+1 / 2} \mathrm{~d} V_{F}-\int v \mathbf{n} \cdot \mathbf{U}^{n+1 / 2} \mathrm{~d} S_{S}=0, \\
& \int v \eta^{n+1} \mathrm{~d} S_{f}=\int v \eta^{n} \mathrm{~d} S_{f}+\Delta t \int \nabla v \cdot \nabla \phi^{n+1 / 2} \mathrm{~d} V_{F}-\Delta t \int v \mathbf{n} \cdot \mathbf{U}^{n+1 / 2} \mathrm{~d} S_{S},
\end{aligned}
$$




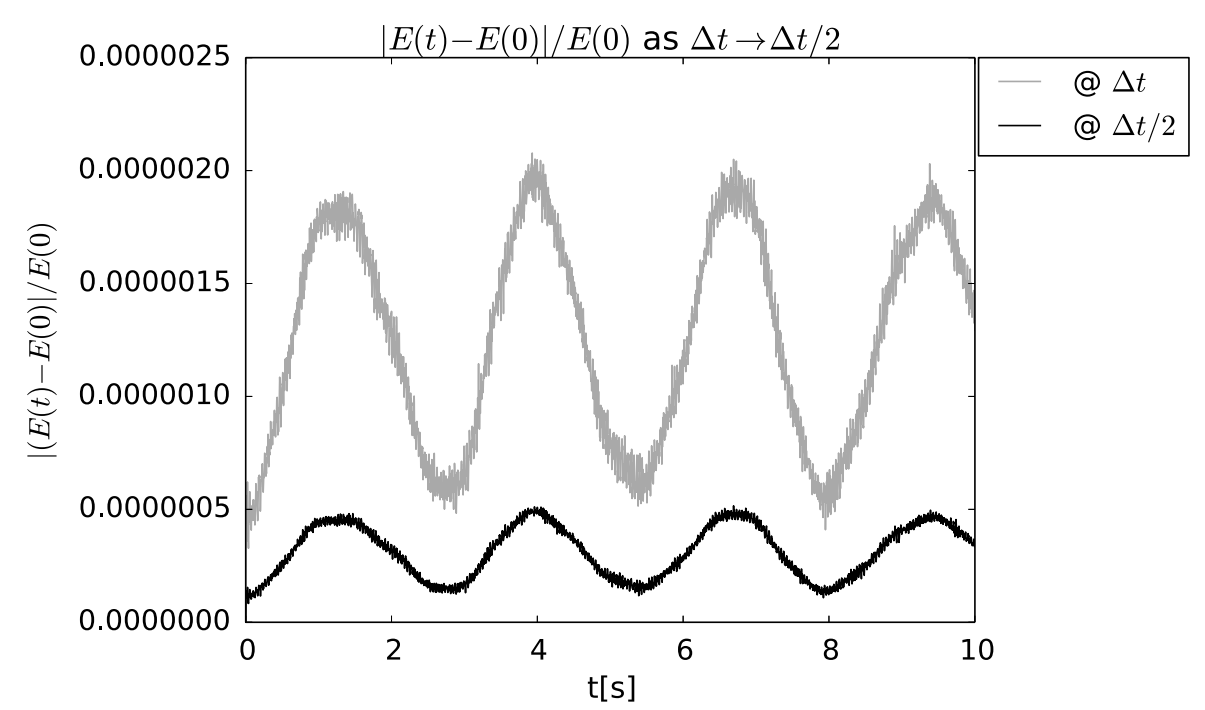

Fig. 7 Convergence of the temporal energy as a function of timestep in 2D: relative-error curves for timesteps $\Delta t$ (upper curve) and $\Delta t / 2$ (lower curve) have amplitudes in the ratio four to one, confirming second-order convergence

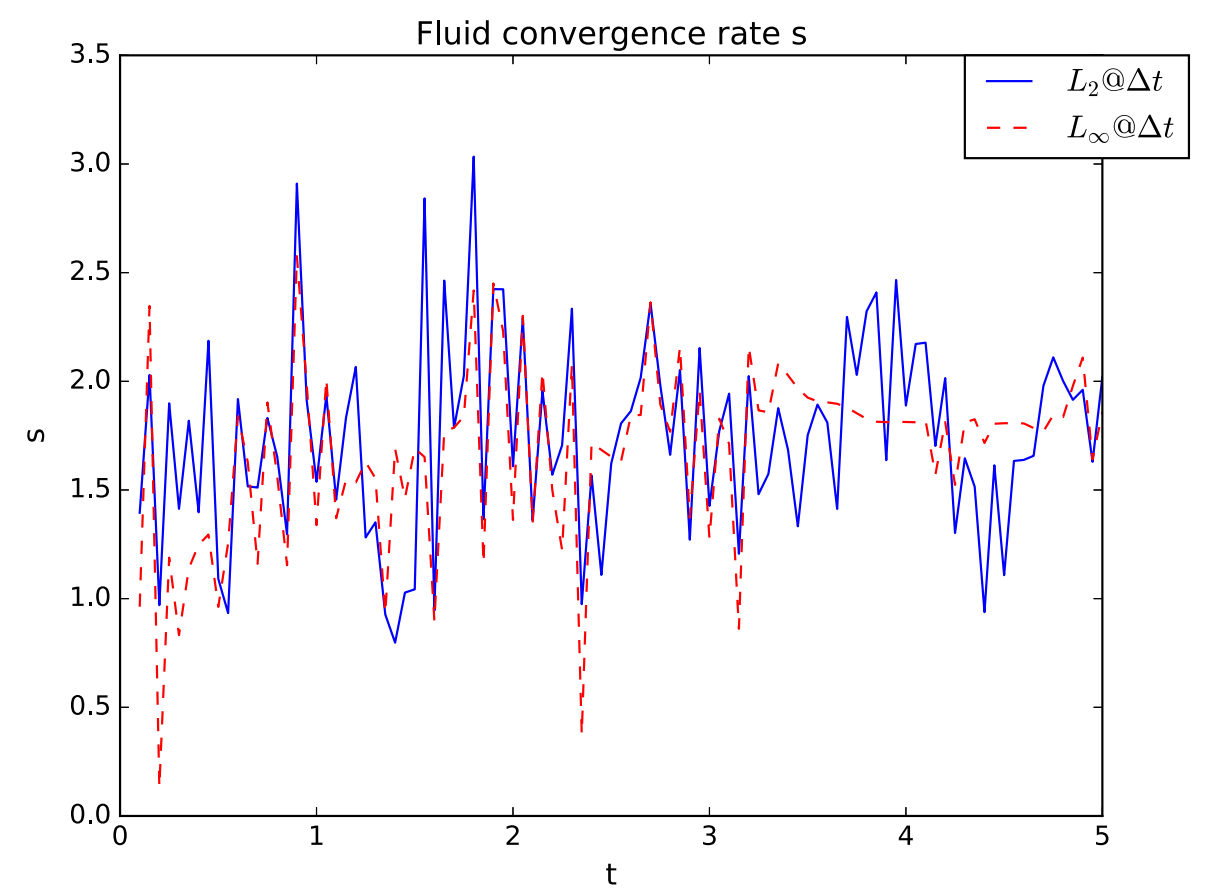

Fig. 8 Rate of convergence, $s$ in (60), of $\phi$ against time, computed using 3 regularly refined meshes and two norms: $L_{2}$ (solid line) and $L_{\infty}$ (dashed line). As the mesh size tends to zero, the theoretical limit of Aitken acceleration yields $s=2$

$$
\begin{aligned}
& \int \mathbf{v} \cdot \mathbf{X}^{n+1} \mathrm{~d} V_{S}=\int \mathbf{v} \cdot\left(\mathbf{X}^{n}+\Delta t \mathbf{U}^{n+1 / 2}\right) \mathrm{d} V_{S} \\
& \int v \phi^{n+1} \mathrm{~d} S_{f}=\int v\left(\phi^{n+1 / 2}-\frac{1}{2} \Delta t \eta^{n+1}\right) \mathrm{d} S_{f} \\
& \int \rho_{0} \mathbf{v} \cdot \mathbf{U}^{n+1} \mathrm{~d} V_{S}+\int \mathbf{n} \cdot \mathbf{v} \phi^{n+1} \mathrm{~d} S_{S}=\rho_{0} \int \mathbf{v} \cdot \mathbf{U}^{n+1 / 2} \mathrm{~d} V_{S},
\end{aligned}
$$


Fig. 9 Temporal snapshots of the 2D water-beam geometry during flow evolution. Although the computational domain is fixed, results have been post-processed into physical space to visualize the deformations. Initial condition of no flow (top) with motion initiated by free-surface displacement. Solutions after $3 \mathrm{~s}$ (middle) and $5 \mathrm{~s}$ (bottom)
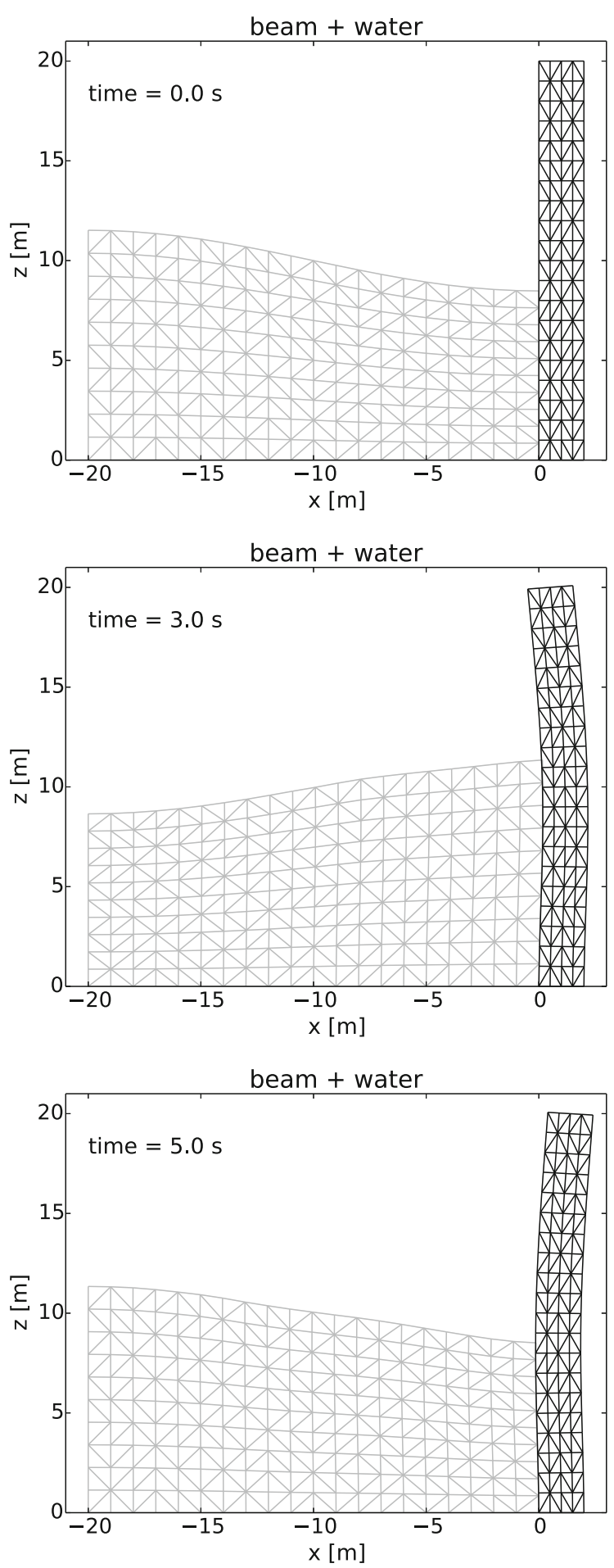
Table 2 Parameter values used in the $3 \mathrm{D}$ computations

\begin{tabular}{lll}
\hline Parameter & Value & Comment \\
\hline$g$ & $9.8 \mathrm{~m} / \mathrm{s}^{2}$ & Gravitational acceleration \\
$L_{x} \times L_{y} \times H_{0}$ & $10 \mathrm{~m} \times 10 \mathrm{~m} \times 4 \mathrm{~m}$ & Water domain \\
$R_{i}$ & $0.6 \mathrm{~m}$ & Beam inner radius \\
$R_{O}$ & $0.8 \mathrm{~m}$ & Beam outer radius \\
$H$ & $12 \mathrm{~m}$ & Beam height \\
$\rho$ & $1000 \mathrm{~kg} / \mathrm{m}^{3}$ & Water density \\
$\rho_{0}$ & $7700 \mathrm{~kg} / \mathrm{m}^{3}$ & Beam density (steel) \\
$\lambda$ & $1 \times 10^{7} \mathrm{~N} / \mathrm{m}^{2}$ & First Lamé constant \\
$\mu$ & $1 \times 10^{7} \mathrm{~N} / \mathrm{m}^{2}$ & Second Lamé constant \\
$N_{z}^{\mathrm{W}}$ & 4 & No. of layers in water \\
$N_{z}^{\mathrm{B}}$ & 12 & No. of layers in beam \\
\hline
\end{tabular}

$$
\begin{gathered}
-\frac{1}{2} \Delta t \int\left(\lambda \nabla \cdot \mathbf{v} \nabla \cdot \mathbf{X}^{n+1}+\mu \partial_{a} X_{b}^{n+1}\left(\partial_{a} v_{b}+\partial_{b} v_{a}\right)\right) \mathrm{d} V_{S}+\int \mathbf{n} \cdot \mathbf{v} \phi^{n+1 / 2} \mathrm{~d} S_{S} \\
\int \nabla v \cdot \nabla \phi^{n+1} \mathrm{~d} V_{F}-\int v \mathbf{n} \cdot \mathbf{U}^{n+1} \mathrm{~d} S_{S}=0
\end{gathered}
$$

in which $\mathrm{d} S_{f}$ denotes integration over the free surface, $\mathrm{d} S_{S}$ the fluid-structure interface, $\mathrm{d} V_{F}$ the fluid domain, $\mathrm{d} V_{S}$ the structure domain, and $\mathbf{n}$ is, as before, the unit outward-normal vector of the fluid domain. In general, the quantities on left-hand side are unknowns. The procedure for solving equations (59) is summarized as follows. The result of (59a) is $\phi^{n+1 / 2}$ at the free surface. It is used as a Dirichlet boundary condition in (59b) and (59c), which are solved simultaneously to get $\phi^{n+1 / 2}$ in the whole fluid domain and $\mathbf{U}^{n+1 / 2}$. Next, $\eta$ is updated in (59d) and $\mathbf{X}$ in (59e). Then (59f) yields $\phi^{n+1}$ at the free surface. Again, it is used as a Dirichlet boundary condition in the simultaneously solved $(59 \mathrm{~g})$ and $(59 \mathrm{~h})$ for the final update of the full $\phi$ and $\mathbf{U}$. In addition, the beam-bottom no-motion boundary condition is applied, i.e., $\mathbf{X}(0, y, z, t)=\mathbf{0}$ in (59e) and $\mathbf{U}(0, y, z, t)=\mathbf{0}$ in (59b), (59c), (59g) and (59h).

The results obtained via the described approach are now presented and discussed.

\section{Results}

Firedrake [9] is an open-source FEM automation package written in python, that uses PETSc for numerical computations. It accepts equations in weak form and automatically assembles the system matrices. Therefore, in this case the scheme in the form (59) was used, with linear continuous Galerkin test functions. For the purposes of illustration and validation, computations were performed first in two dimensions (no $y$-dependence), with bespoke code (no use of Firedrake for automation), constructing directly the matrices in (58). Later, the two-dimensional code in Firedrake gave the same results. Once the scheme was verified to yield a stable solution, computations in three dimensions using Firedrake software were performed.

\subsection{D results}

Parameter values used in this case are shown in Table 1. In order to render visible the beam deformations, Lamé constants are taken to be approximately $10^{4}$ times smaller than those for the steel used to make wind-turbine masts. As previously mentioned, Dirichlet boundary conditions were assumed for the beam, which is fixed (zero displacement 

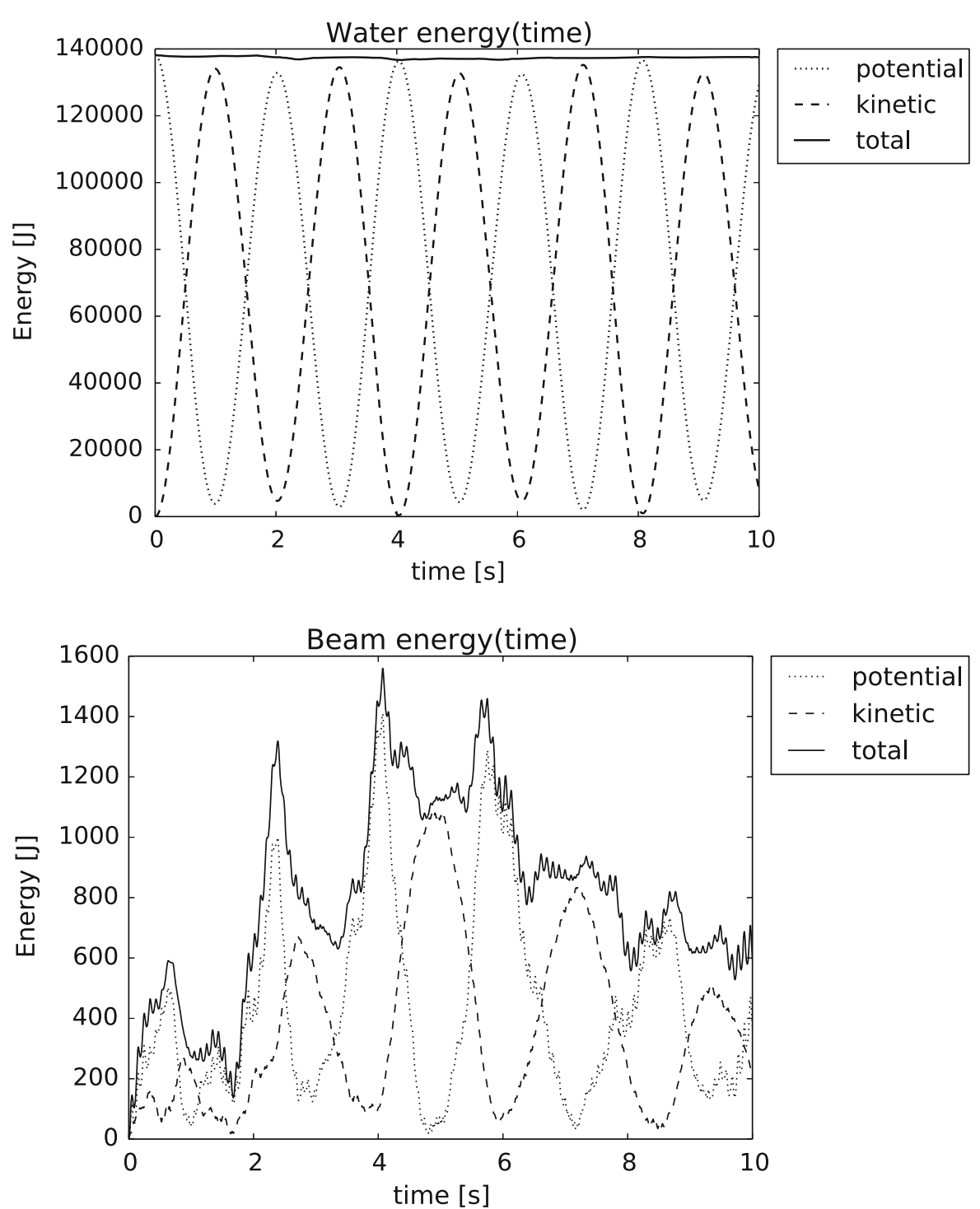

Fig. 10 Energy apportionment (in J) in the 3D system: water (top) and beam (bottom). Curves represent total (continuous), potential (dotted) and kinetic (dashed) energies. Note from the disparate vertical scales in the two plots that the total beam energy is much less than that of the water

and velocity) at its base $z=0$, whereas other boundaries can move freely. We present a solution with zero initial movement and displacement in the beam, and, in the fluid, the first mode of an analytical solution, with deflected initial free surface and no fluid velocity, the natural period of which is $T=5.3 \mathrm{~s}$. The energy in the system is presented in Fig. 6, in which it is clear that, although there is always an energy exchange between the water and beam, the total energy remains constant. As expected, the method is second-order accurate in time, i.e., halving the timestep decreases the difference between the numerically computed energy and the exact one by a factor of four; see Fig. 7 for validation of this convergence. The method is also expected to be second-order accurate in space, as linear basis functions are used in the finite-element expansion. To verify this, we use the formula for the convergence rate derived for the regularly refined-by-halving meshes from Aitken extrapolation:

$s=\log _{2} \frac{\left\|\phi_{\mathrm{f}}-\phi_{\mathrm{m}}\right\|}{\left\|\phi_{\mathrm{m}}-\phi_{\mathrm{c}}\right\|}$ 


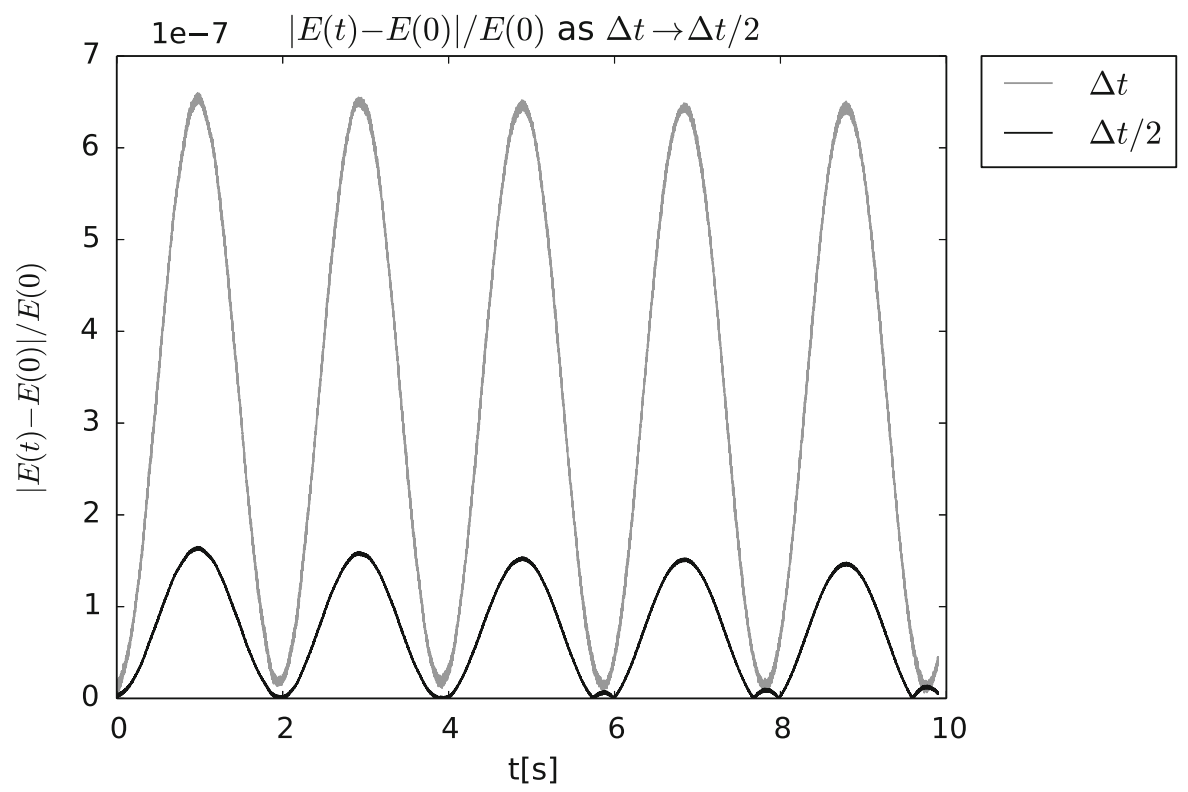

Fig. 11 Convergence of the method as a function of the timestep in 3D: the full timestep (upper curve, grey) and half timestep (lower curve, black) relative-error curves have amplitudes in the ratio four to one, confirming second-order convergence

in which $\phi_{\mathrm{c}}, \phi_{\mathrm{m}}$ and $\phi_{\mathrm{f}}$ are the solutions on coarse, medium and fine meshes, respectively; and $\|\cdot\|$ denotes either the $L_{2}$ or $L_{\infty}$ norm. The convergence rate $s$ computed by (60) is shown in Fig. 8, which shows an oscillatory behaviour around the value of $s=1.7$. Snapshots of the initial condition (no flow, free surface deflected) and evolved state are shown in Fig. 9.

\subsection{D results}

Parameter values for this case are shown in Table 2. The mesh consists of layers of tetrahedra in the $z$-direction, and the fluid domain is asymmetric in the $x y$ plane. The beam is represented by a hollow cylinder, which is meshed with layers of 8 blocks comprising 4 tetrahedra each. Snapshots of the system evolution are shown in Fig. 12. The applied initial condition is one of a beam in equilibrium adjacent to a fluid, free-surface elevation of which is the first mode of a harmonic analytical solution (without the beam) with oscillation period of $4 \mathrm{~s}$. Figure 10 presents the energy transfer in the system. The convergence of the results with decreasing time step is shown in Fig. 11.

\section{Discussion and conclusion}

We have formulated a fully coupled nonlinear variational model of a free-surface fluid-structure interaction. The main benefit is the incorporation of a complex multi-domain, evolving-geometry, single-valued free-surface, transient problem within a unifying and computationally tractable framework with a novel approach to use the Lagrange multiplier $\gamma$ to constrain the beam and fluid common boundary. After elimination of the Lagrange multiplier and the hydrostatic term, the system (28) of linearized water-wave dynamics coupled to an elastic beam, i.e., a system of linearized fluid-structure interaction (FSI) equations, is equivalent to the FSI with the ad hoc coupling derived in [11]. The linear equations have been discretized using a dis/continuous variational FEM, employing techniques from [15], leading to a fully coupled and stable linear FSI with overall energy conservation, i.e., without any energy loss between the subsystems. In the final scheme (58), there appears an extra coupling term in the equation (58d) for the free-surface deviation at the fluid-structure boundary that is not obvious from the continuous equation (28c). 

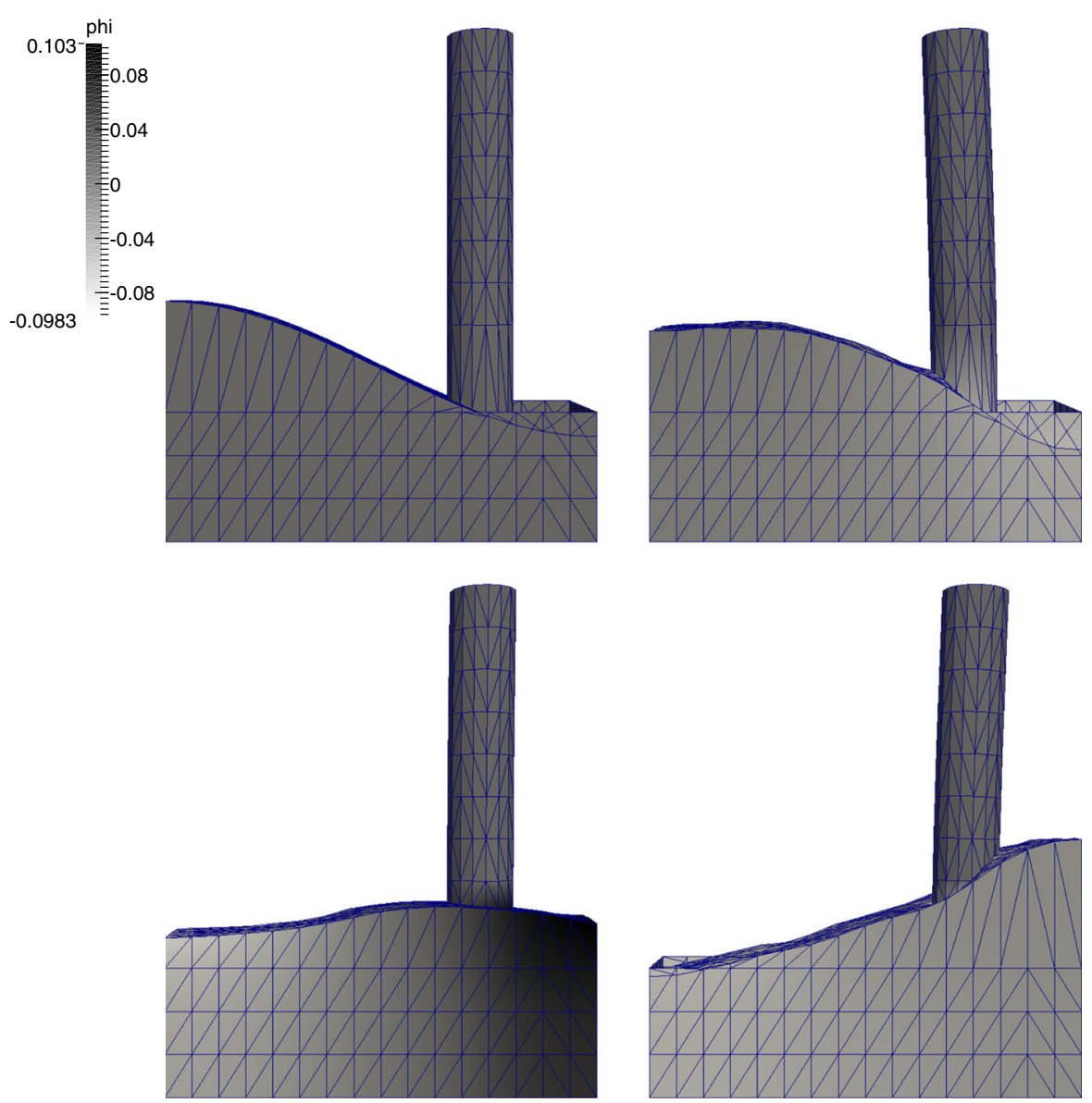

Fig. 12 Temporal snapshots of the 3D water-beam geometry during flow evolution. Although the computational domain is fixed, results have been post-processed into physical space to visualize the deformations. (Top left) Initial condition; no flow; motion initiated by free-surface displacement. Physical flow geometries after $1.1 \mathrm{~s}$ (bottom left), $3.8 \mathrm{~s}$ (top right) and 5.9s (bottom right). Colours, white to black, indicate flow-potential values. A beam deflection is clearly evident

This is a novel aspect that emerges from the variational approach. The numerical extension of these FSI to the nonlinear realm is planned as future research.

The next extension of the model will be to allow for rotational flow to model wave breaking where the free surface can overturn. Non-potential flow and the mixture theory $[1,4]$ of the water-air phase can be used for this purpose. An alternative, which we aim to exploit, is to propose a compressible, Van der Waals-like potential-flow fluid model, that enables the modelling of wave-breaking without actually introducing rotational flow. It is also industrially relevant to look at different models of beam-bottom fixtures, e.g., a flexible spring system instead of a Dirichlet boundary condition. These will comprise future work.

The code used in the 3D computation is available here: https://doi.org/10.5281/zenodo.816221. A simplified 2D version is also published as a tutorial on Firedrake website: http://firedrakeproject.org/demos/linear_fluid_ structure_interaction.py.html.

Acknowledgements This work was supported by the European Commission, Marie Curie Actions - Initial Training Networks (ITN), Project Number 607596. The authors would like to thank Leeds Water Waves group for fruitful discussions and Firedrake developers for their technical support. The authors are also grateful to Ir. Geert Kapsenberg and Dr. Ir. Tim Bunnik from Maritime Research Institute Netherlands (MARIN) for contributing their scientific expertise. 
Open Access This article is distributed under the terms of the Creative Commons Attribution 4.0 International License (http:// creativecommons.org/licenses/by/4.0/), which permits unrestricted use, distribution, and reproduction in any medium, provided you give appropriate credit to the original author(s) and the source, provide a link to the Creative Commons license, and indicate if changes were made.

\section{Appendix A: Linearization of the variational principle}

In this appendix, the details of the linearization of the VP (25) are presented. Some of the terms in (25) can be simplified as follows. First, we consider the term in (25a)

$$
\begin{aligned}
\int_{0}^{L_{s}} & \int_{0}^{L_{y}} \int_{0}^{H_{0}+\eta} \rho \frac{\chi}{L_{s}} \partial_{t} \tilde{x}_{s} \partial_{\chi} \phi-\rho \frac{L_{s}+\tilde{x}_{s}}{L_{s}} \partial_{t} \phi \mathrm{d} z \mathrm{~d} y \mathrm{~d} \chi \mathrm{d} t \\
= & \int_{0}^{L_{s}} \int_{0}^{L_{y}} \int_{0}^{H_{0}+\eta}-\frac{\rho}{L_{s}} \partial_{t} \tilde{x}_{s} \partial_{\chi} \phi+\frac{\rho}{L_{s}} \partial_{t} \tilde{x}_{s} \partial_{\chi} \phi \\
& +\frac{\rho}{L_{s}} \partial_{\chi}(\chi \phi) \partial_{t} \tilde{x}_{s}-\frac{\rho}{L_{s}} \partial_{t}\left(\left(L_{s}+\tilde{x}_{s}\right) \phi\right) \mathrm{d} z \mathrm{~d} y \mathrm{~d} \chi \\
= & \int_{0}^{L_{y}} \int_{0}^{H_{0}+\eta} \rho \phi_{s} \partial_{t} \tilde{x}_{s} \mathrm{~d} z \mathrm{~d} y+\int_{0}^{L_{s}} \int_{0}^{L_{y}} \frac{\rho}{L_{s}}\left(L_{s}+\tilde{x}_{s}\right) \phi_{f} \partial_{t} \eta \mathrm{d} y \mathrm{~d} \chi \\
& -\frac{\mathrm{d}}{\mathrm{d} t} \int_{0}^{L_{s}} \int_{0}^{L_{y}} \int_{0}^{H_{0}+\eta} \frac{\rho}{L_{s}} x_{s} \phi \mathrm{d} z \mathrm{~d} y \mathrm{~d} \chi,
\end{aligned}
$$

in which Leibniz' rule has been used to yield the time derivative of the integral, and then we take the integral of the derivative with respect to $\chi$, thereby obtaining the final term in (61c) as a total time derivative, temporal integration of which, upon using the conditions $\delta \phi(0)=\delta \phi(T)=0$ and $\delta x_{s}(0)=\delta x_{S}(T)=0$, yields a variation in (25) of zero. Therefore, we can neglect this term. We now linearize the remaining two terms in (61c), i.e. neglect terms of the third and higher orders, as quadratic terms in the VP give linear terms in the equations of motion. Thus, the second term in $(61 \mathrm{c})$ becomes

$\int_{0}^{L_{s}} \int_{0}^{L_{y}} \frac{\rho}{L_{s}}\left(L_{s}+\tilde{x}_{s}\right) \phi_{f} \partial_{t} \eta \mathrm{d} y \mathrm{~d} \chi \approx \int_{0}^{L_{s}} \int_{0}^{L_{y}} \rho \phi_{f} \partial_{t} \eta \mathrm{d} y \mathrm{~d} \chi$.

For the first term in (61c), we Taylor-expand around $H_{0}$ to obtain

$\int_{0}^{L_{y}} \int_{0}^{H_{0}+\eta} \rho \phi_{s} \partial_{t} \tilde{x}_{s} \mathrm{~d} z \mathrm{~d} y \approx \int_{0}^{L_{y}} \int_{0}^{H_{0}} \rho \phi_{s} \partial_{t} \tilde{x}_{s} \mathrm{~d} z \mathrm{~d} y$.

The zeroth order of the expansion is sufficient, as the first order already contains cubic terms. We used the definitions of the velocity potentials $\phi_{s}=\phi\left(L_{s}, y, z, t\right)$ and $\phi_{f}=\phi(\chi, y, h(\chi, y, t), t)$, at the beam interface and the free surface, respectively. The first term in (25b) linearizes to

$$
\frac{1}{2} \frac{L_{s}}{x_{s}}\left(\partial_{\chi} \phi\right)^{2}=\frac{1}{2} \frac{1}{1+\tilde{x}_{s} / L_{s}}\left(\partial_{\chi} \phi\right)^{2} \approx \frac{1}{2}\left(1-\frac{\tilde{x}_{s}}{L_{s}}\right)\left(\partial_{\chi} \phi\right)^{2} \approx \frac{1}{2}\left(\partial_{\chi} \phi\right)^{2} .
$$

The second term in (25b) linearizes to

$$
\begin{aligned}
\frac{1}{2} \frac{x_{s}}{L_{s}}\left(-\frac{\chi}{x_{s}} \partial_{y} x_{s} \partial_{\chi} \phi+\partial_{y} \phi\right)^{2} & =\frac{1}{2}\left(\frac{\chi^{2}}{L_{s} x_{s}}\left(\partial_{y} \tilde{x}_{s}\right)^{2}\left(\partial_{\chi} \phi\right)^{2}+\frac{x_{s}}{L_{s}}\left(\partial_{y} \phi\right)^{2}-2 \frac{\chi}{L_{s}} \partial_{y} \tilde{x}_{s} \partial_{z} \phi \partial_{y} \phi\right) \\
& \approx \frac{1}{2} \frac{\chi^{2}}{L_{s}^{2}}\left(\partial_{y} \tilde{x}_{s}\right)^{2}\left(\partial_{z} \phi\right)^{2}+\frac{1}{2}\left(\partial_{y} \phi\right)^{2}-\frac{\chi}{L_{s}} \partial_{y} \tilde{x}_{s} \partial_{z} \phi \partial_{y} \phi \approx \frac{1}{2}\left(\partial_{y} \phi\right)^{2}
\end{aligned}
$$


upon dropping the higher-order terms; a similar linearization occurs for the first term in $(25 \mathrm{c})$. The second term in (25c) linearizes to

$$
\begin{aligned}
& \int_{0}^{L_{s}} \int_{0}^{L_{y}} \int_{0}^{H_{0}+\eta} \rho g\left(z-H_{0}\right)\left(1+\frac{\tilde{x}_{s}}{L_{s}}\right) \mathrm{d} z \mathrm{~d} y \mathrm{~d} \chi \\
& =\int_{0}^{L_{s}} \int_{0}^{L_{y}} \frac{1}{2} \rho g \eta^{2} \mathrm{~d} y \mathrm{~d} \chi-\frac{1}{2} \rho g L_{s} L_{y} H_{0}^{2}+\int_{0}^{L_{s}} \int_{0}^{L_{y}} \int_{0}^{H_{0}+\eta} \rho g\left(z-H_{0}\right) \frac{\tilde{x}_{s}}{L_{s}} \mathrm{~d} z \mathrm{~d} y \mathrm{~d} \chi \\
& \approx \int_{0}^{L_{s}} \int_{0}^{L_{y}} \frac{1}{2} \rho g \eta^{2} \mathrm{~d} y \mathrm{~d} \chi-\frac{1}{2} \rho g L_{s} L_{y} H_{0}^{2}+\int_{0}^{L_{y}} \int_{0}^{H_{0}} \rho g\left(z-H_{0}\right) \tilde{x}_{s} \mathrm{~d} z \mathrm{~d} y,
\end{aligned}
$$

in which third- and higher-order terms have been omitted. The second term in (66c) is a constant and can be dropped, as its variation vanishes. The $-\rho g\left(z-H_{0}\right) \tilde{x}_{s}$ term in $(66 \mathrm{c})$ represents the hydrostatic pressure. Since we are interested in the dynamics of the mutual fluid-structure interaction, we assume that the linearization occurs around an equilibrium state and hence omit the hydrostatic term hereafter. In a similar way, we omit the gravity force term $\rho_{0} g Z$ in (25e), and we use the relations in Sect. 2.3 to simplify the beam expressions. We neglect the subtlety that, in the equilibrium (hydrostatic and lithostatic) state, all $\lambda, \mu$ and $\rho_{0}$ vary slightly along the structure; we assume that they are constant.

Finally, we linearize the Lagrange multiplier $\gamma$ term (25d) by observing that $x_{s}-X=L_{s}+\tilde{x}_{s}-L_{s}-\tilde{X}=\tilde{x}_{s}-\tilde{X}$ and

$$
\begin{aligned}
\tilde{x}_{s}\left(y=Y\left(L_{s}, b, c, t\right), z=Z\left(L_{s}, b, c, t\right), t\right) & =\tilde{x}_{s}\left(y=b+\tilde{Y}\left(L_{s}, b, c, t\right), z=c+\tilde{Z}\left(L_{s}, b, c, t\right), t\right) \\
& =\tilde{x}_{s}(b, c, t)+\left.(\tilde{Y}, \tilde{Z}) \cdot \frac{\partial \tilde{x}_{s}}{\partial(y, z)}\right|_{y=b, z=c}+\cdots
\end{aligned}
$$

In the manipulations in (67), we Taylor-expanded $\tilde{X}$ at the interface around the equilibrium position. $\tilde{X}$ is multiplied by $\gamma$, which, on the other hand, is expanded around zero, since $\gamma=0$ at equilibrium when the hydrostatic pressure is neglected. Therefore, retaining only quadratic terms, the $\gamma$ term (25d) becomes

$$
\begin{aligned}
& \int_{0}^{L_{y}} \int_{0}^{L_{z}} \rho \gamma\left(x_{s}\left(Y\left(L_{s}, b, c, t\right), Z\left(L_{s}, b, c, t\right), t\right)-X\left(L_{s}, b, c, t\right)\right) \mathrm{d} c \mathrm{~d} b \\
& \quad \approx \int_{0}^{L_{y}} \int_{0}^{L_{z}} \rho \gamma\left(\tilde{x}_{s}(b, c, t)-\tilde{X}\left(L_{s}, b, c, t\right)\right) \mathrm{d} c \mathrm{~d} b \\
& \quad \approx \int_{0}^{L_{y}} \int_{0}^{H_{0}} \rho \gamma\left(\tilde{x}_{s}(y, z, t)-\tilde{X}\left(L_{s}, y, z, t\right)\right) \mathrm{d} z \mathrm{~d} y .
\end{aligned}
$$

In (68c), we transformed from Lagrangian to Eulerian coordinates in the linear approximation, as in Sect. 2.3, and the integration in $z$ has been limited to the water height at the structural interface. Higher-order terms arising from the integration from $H_{0}$ to $H_{0}+\eta$ have been neglected. For simplicity of notation, $\chi$ is renamed as $x$ to yield, after incorporating all assumptions, the linearized VP

$$
\begin{aligned}
0= & \delta \int_{0}^{T} \int_{0}^{L_{s}} \int_{0}^{L_{y}} \rho \partial_{t} \eta \phi_{f}-\frac{1}{2} \rho g \eta^{2}-\int_{0}^{H_{0}} \frac{1}{2} \rho|\nabla \phi|^{2} \mathrm{~d} z \mathrm{~d} y \mathrm{~d} x \\
& +\int_{0}^{L_{y}} \int_{0}^{H_{0}} \rho \partial_{t} \tilde{x}_{s} \phi_{s}+\rho \gamma\left(\tilde{x}_{s}(y, z, t)-\tilde{X}\left(L_{s}, y, z, t\right)\right) \mathrm{d} z \mathrm{~d} y \\
& +\int_{L_{s}}^{L_{x}} \int_{0}^{L_{y}} \int_{0}^{L_{z}} \rho_{0} \partial_{t} \tilde{\mathbf{X}} \cdot \mathbf{U}-\frac{1}{2} \rho_{0}|\mathbf{U}|^{2}-\frac{1}{2} \lambda e_{i i} e_{j j}-\mu e_{i j}^{2} \mathrm{~d} z \mathrm{~d} y \mathrm{~d} x \mathrm{~d} t .
\end{aligned}
$$

Due to the linearization, the domain is fixed, and the full system is formulated in Eulerian coordinates. Moreover, the term containing the Lagrange multiplier can be easily removed from (69b) by replacing $\tilde{x}_{s}(y, z, t)$ with $\tilde{X}_{s}=\tilde{X}\left(L_{s}, y, z, t\right)$ elsewhere to obtain the VP without $\gamma$ in the form (27). 


\section{Appendix B: Derivation of temporal discretization}

In this appendix, the details of the derivation of temporal scheme are presented. Given Eq. (57), in terms of original variables, the interim equations of motion arising from the VP for (50) become

$$
\begin{aligned}
& \phi_{\alpha}^{n+1 / 2}=\phi_{\alpha}^{n}-\frac{1}{2} \Delta t \eta_{\alpha}^{n}, \\
& N_{k^{\prime} l^{\prime}}\left(P_{l^{\prime}}^{a}\right)^{n+1 / 2}=N_{k^{\prime} l^{\prime}}\left(P_{l^{\prime}}^{a}\right)^{n}-\frac{1}{2} \Delta t E_{k^{\prime} l^{\prime}}^{a b}\left(X_{l^{\prime}}^{b}\right)^{n}, \\
& M_{\alpha \beta} \eta_{\beta}^{n+1}=M_{\alpha \beta} \eta_{\beta}^{n}+\Delta t\left(B_{\alpha \beta} \phi_{\beta}^{n+1 / 2}-U_{k^{\prime} \alpha}^{a}\left(P_{k^{\prime}}^{a}\right)^{n+1 / 2}\right), \\
& N_{k^{\prime} l^{\prime}}\left(X_{l^{\prime}}^{a}\right)^{n+1}=N_{k^{\prime} l^{\prime}}\left(X_{l^{\prime}}^{a}\right)^{n}+\Delta t\left(-U_{k^{\prime} \alpha}^{a} \phi_{\alpha}^{n+1 / 2}+F_{k^{\prime} l^{\prime}}^{a b}\left(P_{l^{\prime}}^{b}\right)^{n+1 / 2}\right), \\
& \phi_{\alpha}^{n+1}=\phi_{\alpha}^{n+1 / 2}-\frac{1}{2} \Delta t \eta_{\alpha}^{n+1}, \\
& N_{k^{\prime} l^{\prime}}\left(P_{l^{\prime}}^{a}\right)^{n+1}=N_{k^{\prime} l^{\prime}}\left(P_{l^{\prime}}^{a}\right)^{n+1 / 2}-\frac{1}{2} \Delta t E_{k^{\prime} l^{\prime}}^{a b}\left(X_{l^{\prime}}^{b}\right)^{n+1} .
\end{aligned}
$$

The matrices $B, F$ and $U$, appearing in (70) contain the inverse of matrix $C$ which was introduced both to remove the interior $\phi$ degrees of freedom and to reduce the system to the Hamiltonian form. However, once the temporal scheme is obtained, we would like to avoid the costly computation of the inverse of $C$. Therefore, guided by (49), see also [17], we re-introduce $\phi_{i^{\prime}}$ in the interior as

$$
C_{i^{\prime} j^{\prime}} \phi_{i^{\prime}}^{n+1 / 2}=-A_{\alpha j^{\prime}} \phi_{\alpha}^{n+1 / 2}+\left(P_{\widetilde{m}^{\prime}}^{1}\right)^{n+1 / 2} W_{\widetilde{m}^{\prime} n} \delta_{n j^{\prime}}-\delta_{m \alpha} \phi_{\alpha}^{n+1 / 2} \widetilde{m}_{m n} \delta_{n j^{\prime}}
$$

After some manipulations, we find that the final discrete spatiotemporal, fluid-structure interaction equations ( $c f$. (47)) are

$$
\begin{aligned}
& \phi_{\alpha}^{n+1 / 2}=\phi_{\alpha}^{n}-\frac{1}{2} \Delta t \eta_{\alpha}^{n} \\
& N_{k^{\prime} l^{\prime}}\left(P_{l^{\prime}}^{a}\right)^{n+1 / 2}=N_{k^{\prime} l^{\prime}}\left(P_{l^{\prime}}^{a}\right)^{n}-\frac{1}{2} \Delta t E_{k^{\prime} l^{\prime}}^{a b}\left(X_{l^{\prime}}^{b}\right)^{n}, \\
& \left(A_{i^{\prime} j^{\prime}}+\underline{\delta_{i^{\prime} m}} \widetilde{M}_{m n} \delta_{j^{\prime} n}\right) \phi_{i^{\prime}}^{n+1 / 2}=-A_{\alpha j^{\prime}} \phi_{\alpha}^{n+1 / 2}+\underline{\left(\left(P_{\widetilde{m}^{\prime}}^{1}\right)^{n+1 / 2} W_{\widetilde{m}^{\prime} n}-\widetilde{M}_{m n} \phi_{\alpha}^{n+1 / 2} \delta_{\alpha m}\right) \delta_{n j^{\prime}},} \\
& M_{\alpha \beta} \eta_{\beta}^{n+1}=M_{\alpha \beta} \eta_{\beta}^{n}+\Delta t A_{\alpha i} \phi_{i}^{n+1 / 2} \\
& +\underline{\Delta t\left(\phi_{m}^{n+1 / 2} \widetilde{M}_{m n}-\left(P_{\widetilde{m}^{\prime}}^{1}\right)^{n+1 / 2}\right) W_{\widetilde{m}^{\prime} n} \delta_{n \alpha},} \\
& N_{k^{\prime} l^{\prime}}\left(X_{l^{\prime}}^{a}\right)^{n+1}=N_{k^{\prime} l^{\prime}}\left(X_{l^{\prime}}^{a}\right)^{n}+\Delta t N_{k^{\prime} l^{\prime}}\left(P_{l^{\prime}}^{a}\right)^{n+1 / 2}-\underline{\Delta t \delta_{a 1} \delta_{k^{\prime} \widetilde{m}^{\prime}} W_{\widetilde{m}^{\prime} n} \phi_{n}^{n+1 / 2}}, \\
& \phi_{\alpha}^{n+1}=\phi_{\alpha}^{n+1 / 2}-\frac{1}{2} \Delta t \eta_{\alpha}^{n+1} \text {, } \\
& N_{k^{\prime} l^{\prime}}\left(P_{l^{\prime}}^{a}\right)^{n+1}=N_{k^{\prime} l^{\prime}}\left(P_{l^{\prime}}^{a}\right)^{n+1 / 2}-\frac{1}{2} \Delta t E_{k^{\prime} l^{\prime}}^{a b}\left(X_{l^{\prime}}^{b}\right)^{n+1},
\end{aligned}
$$

in which, as in (47), the newly derived coupling terms are underlined.

Implementation of the above formulation leads to a system that conserves energy to second order in the timestep, in keeping with Störmer-Verlet theory. However, using $P$ is inconvenient, as it does not directly represent a physical variable. Moreover, the time evolution of the separate components of (45) reveals an equal and opposite monotonic increase in three terms that involve coupling, which annihilate each other when composed to form the physical energy. This behaviour is possibly related to the fact that not all terms in (45) are positive definite. As a result of this observation, we are motivated to reformulate (72) in terms of the original physical variable (structural velocity),

$U_{k^{\prime}}^{a}=P_{k^{\prime}}^{a}-\delta_{a 1} N_{k^{\prime} \widetilde{m}^{\prime}}^{-1} W_{\widetilde{m}^{\prime} n} \phi_{n}$, 
which is itself motivated by (38) and (43). When this approach is used, the Hamiltonian (45) once more becomes the positive definite (37). Equation (72) is, as a result, amended to the form of (58).

\section{References}

1. Benitz M, Lackner M, Schmidt D (2015) Hydrodynamics of offshore structures with specific focus on wind energy applications. Renew Sustain Energy Rev 44:692-716

2. Bunnik T, Helder J, de Ridder EJ (2015) Simulation of the flexible response of a fixed offshore wind turbine subject to breaking waves. In: 7th international conference on hydroelasticity in marine technology, Split, Croatia, pp 65-81

3. Sagar H, Ley J, el Moctar B (2015) Hydroelasticity effects of wave induced loads on offshore monopile structure. In: 7th international conference on hydroelasticity in marine technology, Split, Croatia pp 82-101

4. Bokhove O, Gidel F, Salwa T (2016) SURFS-UP: Freak waves and breaking wave impact on offshore structures. European industry doctorate mid-term report. Technical report includes description of mixture theory

5. van Daalen E, van Groesen E, Zandbergen P (1993) A Hamiltonian formulation for nonlinear wave-body interactions. In: Eighth international workshop on water waves and floating bodies, IWWWFB, pp 159-163. http://doc.utwente.nl/30984/

6. Luke JC (1967) A variational principle for a fluid with a free surface. J Fluid Mech 27:395-397

7. Miles JW (1977) On Hamilton's principle for surface waves. J Fluid Mech 83:153-158

8. Gagarina E, Ambati V, Nurijanyan S, van der Vegt J, Bokhove O (2016) On variational and symplectic time integrators for Hamiltonian systems. J Comput Phys 306:370-389

9. Rathgeber F, Ham DA., Mitchell L, Lange M, Luporini F, McRae ATT, Bercea G, Markall GR., Kelly PHJ (2016) Firedrake: automating the finite element method by composing abstractions. arXiv:1501.01809 (to appear)

10. Salwa T, Bokhove O, Kelmanson M (2016) Variational coupling of wave slamming against elastic masts. In: 31 st International Workshop on Water Waves and Floating Bodies, IWWWFB, Michigan, pp 149-152. http://www.iwwwfb.org/Abstracts/iwwwfb31/ iwwwfb31_39.pdf

11. Salwa T, Bokhove O, Kelmanson M (2016) Variational modelling of wave-structure interactions for offshore wind turbines. Extended paper for international conference on ocean offshore and arctic engineering, OMAE2016, Busan, South Korea, p 10. http://proceedings.asmedigitalcollection.asme.org/proceeding.aspx?articleID=2570974

12. van Brummelen EH, Shokrpour-Roudbari M, van Zwieten GJ (2016) Elasto-capillarity simulations based on the Navier-StokesCahn-Hilliard equations. Springer, Cham, pp 451-462

13. Hunter S (1976) Mechanics of continuous media. Ellis Horwood, London

14. Leimkuhler B, Reich S (2004) Simulating Hamiltonian dynamics. Cambridge University Press, Cambridge

15. Bokhove O, Kalogirou A (2016) Variational water wave modelling: from continuum to experiment. In: Bridges T, Groves M, Nicholls D (eds) Lectures on the theory of water waves. LMS lecture note series. Cambridge University Press, Cambridge, pp 226-260

16. Huebner B, Walhorn E, Dinkler D (2004) A monolithic approach to fluidstructure interaction using spacetime finite elements. Comput Methods Appl Mech Eng 193(2326):2087-2104

17. Gagarina E, Ambati VR, van der Vegt JJW, Bokhove O (2014) Variational space-time (dis)continuous Galerkin method for nonlinear free surface water waves. J Comput Phys 275:459-483 